\title{
ON A FULLY NONLINEAR YAMABE PROBLEM
}

\author{
By YuXin GE AND GUOFANG WANG
}

Dedicated to Professor W.Y. Ding on the occasion of his 60th birthday

\footnotetext{
ABSTRACT. - We solve the $\sigma_{2}$-Yamabe problem for a nonlocally conformally flat manifold of dimension $n>8$.

(c) 2006 Elsevier Masson SAS
}

RÉSUMÉ. - On résout le problème de $\sigma_{2}$-Yamabe pour des variétés riemanniennes compactes sans bord non localement conformément plates de dimension $n>8$.

(c) 2006 Elsevier Masson SAS

\section{Introduction}

Let $\left(M, g_{0}\right)$ be a compact, oriented Riemannian manifold with metric $g_{0}$ and $\left[g_{0}\right]$ the conformal class of $g_{0}$. Let $\operatorname{Ric}_{g}$ and $R_{g}$ be the Ricci tensor and scalar curvature of $g$ respectively. The Schouten tensor of the metric $g$ is defined by

$$
S_{g}=\frac{1}{n-2}\left(\operatorname{Ric}_{g}-\frac{R_{g}}{2(n-1)} \cdot g\right)
$$

The Schouten tensor plays an important role in conformal geometry. Let $\sigma_{k}$ be the $k$ th elementary symmetric function. For a symmetric $n \times n$ matrix $A$, set $\sigma_{k}(A)=\sigma_{k}(\Lambda)$, where $\Lambda=\left(\lambda_{1}, \lambda_{2}, \ldots, \lambda_{n}\right)$ is the set of eigenvalues of $A$. The $\sigma_{k}$-scalar curvature of $g$ is defined by

$$
\sigma_{k}(g):=\sigma_{k}\left(g^{-1} \cdot S_{g}\right),
$$

where $g^{-1} \cdot S_{g}$ is locally defined by $\left(g^{-1} \cdot S_{g}\right)_{j}^{i}=\sum_{k} g^{i k}\left(S_{g}\right)_{k j}$, see [38]. Note that $\sigma_{1}(g)=$ $\frac{1}{2(n-1)} R_{g}$. It is an interesting question to find a metric $g$ in a given conformal class $\left[g_{0}\right]$ such that

$$
\sigma_{k}(g)=c
$$

for some constant $c$. Since the Schouten tensors $S_{g}$ and $S_{g_{0}}$ of conformal metrics $g=e^{-2 u} g_{0}$ and $g_{0}$ have the following relation

$$
S_{g}=\nabla^{2} u+d u \otimes d u-\frac{|\nabla u|^{2}}{2} g_{0}+S_{g_{0}}
$$

ANNALES SCIENTIFIQUES DE L'ÉCOLE NORMALE SUPÉRIEURE 0012-9593/04/@ 2006 Elsevier Masson SAS. All rights reserved. 
Eq. (1) is equivalent to the following fully nonlinear equation

$$
\sigma_{k}\left(\nabla^{2} u+d u \otimes d u-\frac{|\nabla u|^{2}}{2} g_{0}+S_{g_{0}}\right)=c e^{-2 k u},
$$

for some constant $c$. When $k=1$, it is the well-known Yamabe equation.

Let

$$
\Gamma_{k}^{+}=\left\{\Lambda=\left(\lambda_{1}, \lambda_{2}, \ldots, \lambda_{n}\right) \in \mathbb{R}^{n} \mid \sigma_{j}(\Lambda)>0, \forall j \leqslant k\right\}
$$

be Gårding's cone. A metric $g$ is said to be $k$-positive or simply $g \in \Gamma_{k}^{+}$if $\sigma_{j}(g)(x)>0$ for any $j \leqslant k$ and at every point $x \in M$. If $g=e^{-2 u} g_{0}$, we say $u$ is $k$-admissible if $g$ is $k$-positive. In this paper we consider the following

$\sigma_{k}$-Yamabe problem. - Let $g_{0} \in \Gamma_{k}^{+}$. Find a conformal metric $g \in\left[g_{0}\right] \cap \Gamma_{k}^{+}$such that

$$
\sigma_{k}(g)=c
$$

for some constant $c$.

The study of the fully nonlinear equations (1) was initiated by Viaclovsky. Since then there is a lot of work concerning these equations. Here, we just mention some results directly related to the existence of the $\sigma_{k}$-Yamabe problem. This problem has been solved in the following cases. When $k=n$, under a sufficient condition, Viaclovsky proved the existence in [40]. When $n=2 k=4$, which is an important case, Chang-Gursky-Yang solved the problem in [7]. See also [6,23]. When the underlying manifold is locally conformally flat, this problem was solved by Guan and Wang [19] and $\mathrm{Li}$ and $\mathrm{Li}$ [29] independently. See also [4]. Note that when the underlying manifold $\left(M, g_{0}\right)$ is locally conformally flat and $g \in \Gamma_{k}^{+}$with $k \geqslant n / 2$, the universal cover of $M$ is conformally equivalent to a spherical space form [17]. When $k>n / 2$, the $\sigma_{k}$-Yamabe problem was solved by Gursky-Viaclovsky in [24]. See also their earlier work [22].

In this paper, we consider the case $k=2$. In this case, Eq. (2) is a variational problem, which was observed by Viaclovsky in [38]. This is crucial for our method presented here. Our main result in this paper is

THEOREM 1. - Let $\left(M^{n}, g_{0}\right)$ be a compact, oriented Riemannian manifold with $g_{0} \in \Gamma_{2}^{+}$. When $n>8$ and the Weyl tensor $W_{g_{0}} \neq 0$, then there is a conformal metric $g \in\left[g_{0}\right] \cap \Gamma_{2}^{+}$such that

$$
\sigma_{2}(g)=c
$$

for some constant $c$.

Combining the results of $[19,29]$, the $\sigma_{2}$-Yamabe problem is solvable if $n>8$. Like the ordinary Yamabe problem, there is a well-known difficulty-the loss of compactness of Eq. (1). Another more difficult problem is the fully nonlinearity of (1). Our result here is an analogue of the result of Aubin [2] for the ordinary Yamabe problem. Even the ideas of proof are quite similar. However the techniques to realize these ideas become more delicate due to the fully nonlinearity.

Set $\mathcal{C}_{2}=\left\{g \in\left[g_{0}\right] \mid g \in \Gamma_{2}^{+}\right\}$and define a Yamabe type constant by

$$
Y_{2}\left(M,\left[g_{0}\right]\right)= \begin{cases}\inf _{g \in \mathcal{C}_{2}} \widetilde{\mathcal{F}}_{2}(g), & \text { if } \mathcal{C}_{2} \neq \emptyset, \\ +\infty, & \text { if } \mathcal{C}_{2}=\emptyset,\end{cases}
$$

$4^{\text {e }}$ SÉRIE - TOME $39-2006-\mathrm{N}^{\circ} 4$ 
where $\widetilde{\mathcal{F}}_{2}(g)=\operatorname{vol}(g)^{-\frac{n-4}{n}} \int_{M} \sigma_{2}(g) d \operatorname{vol}(g)$. This is a natural generalization of the Yamabe constant that was considered in [20] in the fully nonlinear context.

We first prove the following proposition.

Proposition 1. - Let $\left(M^{n}, g_{0}\right)$ be a compact, oriented Riemannian manifold of dimension $n>4$ with $g_{0} \in \Gamma_{2}^{+}$. The $\sigma_{2}$-Yamabe problem is solvable, provided that

$$
Y_{2}\left(M,\left[g_{0}\right]\right)<Y_{2}\left(\mathbb{S}^{n}\right) .
$$

The idea to prove the proposition is a "blow-up" analysis, which is a typical tool in the field of semilinear equations. The observation that the fully nonlinear equation (1) also admits a blow-up analysis was made in [18]. Inspired by Yamabe's approach (see e.g. [42,3]), we first prove the existence of solutions to a "subcritical" equation (6) for any small $\varepsilon>0$. To prove the existence of solutions of (6), we use a fully nonlinear flow (8). We show that this flow globally converges to a solution $u_{\varepsilon}$ of the subcritical equation (6). In fact, $u_{\varepsilon}$ is a minimizer for a corresponding functional. This is one of the crucial points of this paper. Then we consider the sequence $u_{\varepsilon}$ as $\varepsilon \rightarrow 0$. Using the blow-up analysis developed in [18] and the classification of "bubbles" in [8] or [29], we can show that the sequence $u_{\varepsilon}$ subconverges to a solution of (2) under the condition (3). The flow method to attack the existence of fully nonlinear equations was used by many mathematicians, see for instance $[9,41,37,10]$. In the fully nonlinear conformal equations, it was used in $[19,20]$.

Then we show

PROPOSITION 2. - Let $\left(M^{n}, g_{0}\right)$ be a compact, oriented Riemannian manifold with $g_{0} \in \Gamma_{2}^{+}$. When $n>8$ and the Weyl tensor $W_{g_{0}} \neq 0$,

$$
Y_{2}\left(M,\left[g_{0}\right]\right)<Y_{2}\left(\mathbb{S}^{n}\right) .
$$

The proof of this proposition is a delicate gluing argument. We need to construct suitable test metrics as in $[2,32]$ for the ordinary Yamabe problem. A subtle point in the gluing is that all metrics we constructed should lie in $\Gamma_{2}^{+}$. Recall that in the ordinary Yamabe problem, the test metrics constructed by Aubin and Schoen have negative scalar curvature somewhere. To overcome this difficulty, we adopt a method of Gromov-Lawson in their construction of metrics of positive scalar curvature. A similar method was also used in [31] for metrics of positive isotropic curvature and [15] for metrics of positive $\Gamma_{k}$-curvatures on locally conformally flat manifolds. See also $[25,33]$. We believe that by a similar, but more delicate construction one can prove Proposition 2 for $n=8$. For $n=5,6,7$, this problem becomes delicate. We will consider these cases later.

By-products of our work for flow (8) are the Poincaré type inequality and Sobolev inequality for the operator $\sigma_{2}\left(\nabla^{2} u+d u \otimes d u-\frac{|\nabla u|^{2}}{2} g_{0}+S_{g_{0}}\right)$. (In Section 4, we provide another proof.)

Proposition 3. - Let $\left(M, g_{0}\right)$ be a compact, oriented Riemannian manifold with $g_{0} \in \Gamma_{2}^{+}$ and the dimension $n>4$. Then there exists a positive constant $\lambda_{1}>0$ depending only on $\left(M, g_{0}\right)$ such that for any $C^{2}$ function $u$ with $e^{-2 u} g_{0} \in \mathcal{C}_{2}\left(\left[g_{0}\right]\right)$ we have

$$
\int_{M} \sigma_{2}\left(e^{-2 u} g_{0}\right) d \operatorname{vol}\left(e^{-2 u} g_{0}\right) \geqslant \lambda_{1} \int e^{4 u} d \operatorname{vol}\left(e^{-2 u} g_{0}\right) \text {. }
$$

Equivalently, for such a function $u$ we have

$$
\int_{M} e^{(4-n) u} \sigma_{2}\left(\nabla^{2} u+d u \otimes d u-\frac{|\nabla u|^{2}}{2} g_{0}+S_{g_{0}}\right) d \operatorname{vol}\left(g_{0}\right) \geqslant \lambda_{1} \int e^{(4-n) u} d \operatorname{vol}\left(g_{0}\right) .
$$


THEOREM 2. - Let $\left(M, g_{0}\right)$ be a compact, oriented Riemannian manifold with $g_{0} \in \Gamma_{2}^{+}$and the dimension $n>4$. Then there exists a positive constant $C>0$ depending only on $\left(M, g_{0}\right)$ such that for any $C^{2}$ function $u$ with $e^{-2 u} g_{0} \in \mathcal{C}_{2}\left(\left[g_{0}\right]\right)$ we have

$$
\int_{M} \sigma_{2}\left(e^{-2 u} g_{0}\right) d \operatorname{vol}\left(e^{-2 u} g_{0}\right) \geqslant C \operatorname{vol}\left(e^{-2 u} g_{0}\right)^{\frac{n-4}{n}} .
$$

Equivalently, for such a function $u$ we have

$$
\int_{M} e^{(4-n) u} \sigma_{2}\left(\nabla^{2} u+d u \otimes d u-\frac{|\nabla u|^{2}}{2} g_{0}+S_{g_{0}}\right) d \operatorname{vol}\left(g_{0}\right) \geqslant C\left(\int_{M} e^{-n u} d \operatorname{vol}\left(g_{0}\right)\right)^{\frac{n-4}{n}} .
$$

The Sobolev inequality and other geometric inequalities, the Moser-Trudinger inequality and a conformal quermassintegral inequality for $\sigma_{k}\left(\nabla^{2} u+d u \otimes d u-\frac{|\nabla u|^{2}}{2} g_{0}+S_{g_{0}}\right)$ for a locally conformally flat manifold were established in $[20]$. See also $[17,13]$.

The method presented here works for a conformal quotient equation

$$
\frac{\sigma_{2}(g)}{\sigma_{1}(g)}=c,
$$

on a general manifold [12]. See other results for conformal quotient equations in [20,16,24].

The paper is organized as follows. In Section 2, we discuss various fully nonlinear flows and we prove local estimates for these flows in Section 3. In Section 4, we establish the Poincaré and Sobolev inequalities. We prove the global convergence of these fully nonlinear flows and Proposition 1 in Section 5. In Section 6, we prove Proposition 2, and hence Theorem 1.

\section{Various flows and ideas of proof}

Consider the following functional

$$
\mathcal{F}_{k}(g)=\int_{M} \sigma_{k}(g) d \operatorname{vol}(g)
$$

and its normalization $\widetilde{\mathcal{F}}_{k}$

$$
\widetilde{\mathcal{F}}_{k}(g)=\operatorname{vol}(g)^{-\frac{n-2 k}{n}} \int_{M} \sigma_{k}(g) d \operatorname{vol}(g) .
$$

When $k=2$ or the underlying manifold is locally conformally flat, Viaclovsky proved that critical points of $\widetilde{\mathcal{F}}_{2}$ are solutions of (1). Therefore, in these cases, (1) is a variational problem. The case when the underlying manifold is locally conformally flat was studied in [19,29], as mentioned in the introduction. See also [4]. In this paper we only consider the case $k=2$. Since the case $k=2$ and $n \leqslant 4$ was solved in [6,22,24], we focus on the case $k=2$ and $n>4$.

Recall that $\mathcal{C}_{2}=\left\{g \in\left[g_{0}\right] \mid g \in \Gamma_{2}^{+}\right\}$and the Yamabe type constant is defined by

$$
Y_{2}\left(M,\left[g_{0}\right]\right)= \begin{cases}\inf _{g \in \mathcal{C}_{2}} \widetilde{\mathcal{F}}_{2}(g), & \text { if } \mathcal{C}_{2} \neq \emptyset, \\ \infty, & \text { if } \mathcal{C}_{2}=\emptyset\end{cases}
$$

$4^{\mathrm{e}}$ SÉRIE - TOME $39-2006-\mathrm{N}^{\circ} 4$ 
Our main aim of this paper is to show that $Y_{2}\left(M,\left[g_{0}\right]\right)$ is achieved for nonlocally conformally flat manifolds when $\mathcal{C}_{2}\left(\left[g_{0}\right]\right) \neq \emptyset$. In order to achieve our aim, we will first consider subcritical equations

$$
\sigma_{2}^{1 / 2}\left(\nabla^{2} u+d u \otimes d u-\frac{|\nabla u|^{2}}{2} g_{0}+S_{g_{0}}\right)=c e^{(\varepsilon-2) u},
$$

for $\varepsilon \in(0,2]$ and the positive constant $c$. Its corresponding functional is

$$
\widetilde{\mathcal{F}}_{2, \varepsilon}(g)=V_{\varepsilon}(g)^{-\frac{n-4}{n-2 \varepsilon}} \int_{M} \sigma_{2}(g) d \operatorname{vol}(g),
$$

where

$$
V_{\varepsilon}(g):=\int_{M} e^{2 \varepsilon u} d \operatorname{vol}(g)=\int_{M} e^{(2 \varepsilon-n) u} d \operatorname{vol}\left(g_{0}\right),
$$

for $g=e^{-2 u} g_{0}$. It is clear that $V_{0}(g)=\operatorname{vol}(g)$, the volume of $g$ and $V_{2}(g)=\int e^{(4-n) u} d \operatorname{vol}(g)$. Set

$$
Y_{\varepsilon}\left(M,\left[g_{0}\right]\right)=\inf _{g \in \mathcal{C}_{2}} \widetilde{\mathcal{F}}_{2, \varepsilon}(g) .
$$

We will show that $Y_{\varepsilon}\left(M,\left[g_{0}\right]\right)$ is achieved at $u_{\varepsilon}$, which is clearly a solution of (6). To prove this we consider the following fully nonlinear flow

(8) $2 \frac{d u}{d t}=-g^{-1} \cdot \frac{d}{d t} g$

$$
\begin{aligned}
& =\left(h\left(e^{-2 u} \sigma_{2}^{1 / 2}(g)\right)-h\left(r_{\varepsilon}^{1 / 2}(g) e^{(\varepsilon-2) u}\right)\right)-s_{\varepsilon}(g) \\
& =h\left(\sigma_{2}^{1 / 2}\left(\nabla^{2} u+d u \otimes d u-\frac{|\nabla u|^{2}}{2} g_{0}+S_{g_{0}}\right)\right)-h\left(r_{\varepsilon}^{1 / 2}(g) e^{(\varepsilon-2) u}\right)-s_{\varepsilon}(g),
\end{aligned}
$$

with initial value $u(0)=1$, where $r_{\varepsilon}(g)$ and $s_{\varepsilon}(g)$ are given by for any $\varepsilon \in[0,2]$

$$
\begin{aligned}
r_{\varepsilon}(g) & :=\frac{\int_{M} \sigma_{2}(g) d \operatorname{vol}(g)}{\int_{M} e^{2 \varepsilon u} d \operatorname{vol}(g)} \\
s_{\varepsilon}(g) & :=\frac{\int_{M} e^{2 \varepsilon u}\left(h\left(e^{-2 u} \sigma_{2}^{1 / 2}(g)\right)-h\left(r_{\varepsilon}^{1 / 2}(g) e^{(\varepsilon-2) u}\right)\right) d \operatorname{vol}(g)}{\int_{M} e^{2 \varepsilon u} d \operatorname{vol}(g)}
\end{aligned}
$$

and $h: \mathbb{R}_{+} \rightarrow \mathbb{R}$ is smooth concave function with $h^{\prime}(t) \geqslant 1$ for $t \in \mathbb{R}_{+}$satisfying

$$
h(s)= \begin{cases}2 \log s & \text { if } t \leqslant 1 \\ s & \text { if } t \geqslant 2 .\end{cases}
$$

Flow (8) preserves $V_{\varepsilon}$ and nonincreases $\mathcal{F}_{2}$.

LEMma 1. - For any $\varepsilon \in[0,2]$, the flow (8) preserves the functional $V_{\varepsilon}$ and nonincreases $\mathcal{F}_{2}$. In fact, we have

$$
\frac{d}{d t} \mathcal{F}_{2}(g)=-\frac{n-4}{2} \int_{M}\left(h\left(e^{-2 u} \sigma_{2}^{1 / 2}(g)\right)-h\left(r_{\varepsilon}^{1 / 2}(g) e^{(\varepsilon-2) u}\right)\right)\left(\sigma_{2}(g)-r_{\varepsilon} e^{2 \varepsilon u}\right) d \operatorname{vol}(g) .
$$

Moreover, $r_{\varepsilon}$ is bounded. 
Proof. - We note that

$$
\frac{d}{d t} \mathcal{F}_{2}(g)=\frac{n-4}{2} \int_{M}\left(g^{-1} \cdot \frac{d}{d t} g\right) \sigma_{2}(g) d \operatorname{vol}(g)
$$

and

$$
\frac{d}{d t} V_{\varepsilon}(g)=\frac{n-2 \varepsilon}{2} \int_{M}\left(g^{-1} \cdot \frac{d}{d t} g\right) e^{2 \varepsilon u} d \operatorname{vol}(g)=0 .
$$

See the proof in [19]. It is clear that $V_{\varepsilon}$ is preserved along the flow. On the other hand, a direct computation gives

$$
\begin{aligned}
\frac{d}{d t} \mathcal{F}_{2}(g)= & \frac{n-4}{2} \int_{M}\left(g^{-1} \cdot \frac{d}{d t} g\right)\left(\sigma_{2}(g)-r_{\varepsilon} e^{2 \varepsilon u}\right) d \operatorname{vol}(g) \\
= & -\frac{n-4}{2} \int_{M}\left(h\left(e^{-2 u} \sigma_{2}^{1 / 2}(g)\right)-h\left(r_{\varepsilon}^{1 / 2}(g) e^{(\varepsilon-2) u}\right)\right) \\
& \times\left(\sigma_{2}(g)-r_{\varepsilon} e^{2 \varepsilon u}\right) d \operatorname{vol}(g),
\end{aligned}
$$

where in the second equality we have used the fact

$$
\int_{M}\left(\sigma_{2}(g)-r_{\varepsilon} e^{2 \varepsilon u}\right) d \operatorname{vol}(g)=0 .
$$

Now it is easy to see that $r_{\varepsilon}$ is bounded.

In fact, flow (8) strictly decreases the functional $\mathcal{F}_{2}$ except at the solutions of Eq. (6). When $\varepsilon=0$ Eq. (6) is just (2). When $\varepsilon=2$ Eq. (6) is a corresponding equation for a nonlinear eigenvalue problem, which was considered in [21]. See also Section 4.

Since $g_{0} \in \Gamma_{2}^{+}$, flow (8) is parabolic near $t=0$. By the standard implicit function theorem we have the following short-time existence result.

Proposition 4. - For any $g_{0} \in C^{\infty}(M)$ with $g_{0} \in \Gamma_{2}^{+}$, there exists a positive constant $T^{*} \in(0, \infty]$ such that flow (8) exists and is parabolic for $t \in\left[0, T^{*}\right)$, and $\forall T<T^{*}$,

$$
g \in C^{3, \alpha}([0, T] \times M), \quad \forall 0<\alpha<1, \quad \text { and } \quad g(t) \in \Gamma_{2}^{+} .
$$

We assume that $T^{*}$ is the largest number, for which Proposition 4 holds. We first show the global convergence of flow (8) when $\varepsilon=2$. The global convergence implies a Poincaré type inequality. Then, using this inequality and the divergence free of the first Newton transformation of the Schouten tensor, which was an observation due to Viaclovsky, we obtain an optimal Sobolev inequality. By establishing a flow version of local gradient estimates, which was proved in [18], we show that flow (8) globally converges to a solution $u_{\varepsilon}$ of (6) for any $\varepsilon \in(0,2]$. With the help of the local estimate obtained in [18] and a classification in [29] or [8], we show that $u_{\varepsilon}$ subconverges to a solution $u_{0}$ of (2), provided that

$$
Y_{2}\left(M,\left[g_{0}\right]\right)<Y_{2}\left(\mathbb{S}^{n}\right) .
$$

In this case, it is clear that $u_{0}$ is the minimum of $\widetilde{\mathcal{F}}_{2}$. 


\section{Local estimates}

In this section, we will establish a local estimate for solutions of (8), which is a parabolic version of a local estimate for solutions of (2) obtained in [18].

THEOREM 3. - Let $u$ be a solution of (8) with $\varepsilon \in[0,2]$ in a geodesic ball $B_{r} \times[0, T]$ for $T<T^{*}$ and $r<r_{0}$, the injectivity radius of $M$. There is a constant $C>0$ depending only on $\left(B_{r}, g_{0}\right)$ such that for any $(x, t) \in B_{r / 2} \times[0, T]$

$$
|\nabla u|^{2}+\left|\nabla^{2} u\right| \leqslant C\left(1+e^{-(2-\varepsilon) \inf _{(x, t) \in B_{r} \times[0, T]} u(x, t)}\right) .
$$

Proof. - The proof follows [18] closely. We only point out the different places. Without loss of generality, we assume $r=1$. Let $\rho \in C_{0}^{\infty}\left(B_{1}\right)$ be a test function defined as in [18] such that

$$
\begin{array}{clrl}
\rho \geqslant 0, & & \text { in } B_{1}, \\
\rho=1, & & \text { in } B_{1 / 2}, \\
|\nabla \rho(x)| \leqslant 2 b_{0} \rho^{1 / 2}(x), & & \text { in } B_{1}, \\
\left|\nabla^{2} \rho\right| \leqslant b_{0}, & & \text { in } B_{1} .
\end{array}
$$

Here $b_{0}>1$ is a constant. Set $H(x, t)=\rho|\nabla u|^{2}$. Let $\left(x_{0}, t_{0}\right)$ be the maximum of $H$ in $M \times[0, T]$. Without loss of generality, we assume $t_{0}>0$. We have at $\left(x_{0}, t_{0}\right)$ that

$$
\begin{aligned}
& 0 \leqslant H_{t}=2 \rho \sum_{l} u_{l} u_{l t}, \\
& 0=H_{j}=\rho_{j}|\nabla u|^{2}+2 \rho \sum_{l} u_{l} u_{l j}, \\
& 0 \geqslant\left(H_{i j}\right) .
\end{aligned}
$$

Let $W=\left(w_{i j}\right)$ be an $n \times n$ matrix with $w_{i j}=\nabla_{i j}^{2} u+u_{i} u_{j}-\frac{|\nabla u|^{2}}{2}\left(g_{0}\right)_{i j}+\left(S_{g_{0}}\right)_{i j}$. Here $u_{i}$ and $u_{i j}$ are the first and second derivatives of $u$ with respect to the background metric $g_{0}$. By choosing suitable normal coordinates, we may assume that $W$ is diagonal at $\left(x_{0}, t_{0}\right)$, and hence we have at $\left(x_{0}, t_{0}\right)$,

$$
\begin{aligned}
& w_{i i}=u_{i i}+u_{i}^{2}-\frac{1}{2}|\nabla u|^{2}+\left(S_{g_{0}}\right)_{i i}, \\
& u_{i j}=-u_{i} u_{j}-\left(S_{g_{0}}\right)_{i j}, \quad \forall i \neq j .
\end{aligned}
$$

In view of (13), (15) and (17), we have at $\left(x_{0}, t_{0}\right)$

$$
\left|\sum_{l=1}^{n} u_{i l} u_{l}\right| \leqslant b_{0} \rho^{-1 / 2}|\nabla u|^{2} .
$$

We may assume that

$$
H\left(x_{0}, t_{0}\right) \geqslant A_{0}^{2} b_{0}^{2}
$$

i.e., $\rho^{-1 / 2} \leqslant \frac{1}{A_{0} b_{0}}|\nabla u|$, and

$$
\left|\nabla S_{g_{0}}\right|+\left|S_{g_{0}}\right| \leqslant A_{0}^{-1}|\nabla u|^{2}
$$


where $A_{0}>1$ is a large, but fixed number to be chosen later, otherwise we are done. Thus, from (18) we have

$$
\left|\sum_{l=1}^{n} u_{i l} u_{l}\right| \leqslant \frac{|\nabla u|^{3}}{A_{0}}\left(x_{0}, t_{0}\right) .
$$

Set $F=h\left(\sigma_{2}^{1 / 2}(W)\right)$ and

$$
F^{i j}=\frac{\partial F(W)}{\partial w_{i j}} .
$$

Note that flow (8) is equivalent to $2 u_{t}=F-h\left(r_{\varepsilon}^{1 / 2} e^{(\varepsilon-2) u}\right)-s_{\varepsilon}$ and $F^{i j}$ is diagonal at $\left(x_{0}, t_{0}\right)$. Since matrix $\left(F^{i j}\right)$ is positive definite, from (14) and (16) we have

$$
\begin{aligned}
0 \geqslant & \sum_{i, j} F^{i j} H_{i j}-2 H_{t} \\
= & \sum_{i, j} F^{i j}\left\{\left(-2 \frac{\rho_{i} \rho_{j}}{\rho}+\rho_{i j}\right)|\nabla u|^{2}+2 \rho \sum_{l} u_{l i j} u_{l}+2 \rho \sum_{l} u_{i l} u_{j l}\right\} \\
& -4 \rho \sum_{l} u_{l} u_{l t} .
\end{aligned}
$$

We need to estimate the term $\sum_{i, j, l} F^{i j} u_{l i j} u_{l}-2 \sum_{l} u_{l} u_{l t}$. Since changing the order of derivatives only causes a low order term, we have

$$
\begin{aligned}
& \sum_{i, j, l} F^{i j} u_{l i j} u_{l}-2 \sum_{l} u_{l} u_{l t} \\
& \geqslant \sum_{i, j, l} F^{i j} u_{i j l} u_{l}-2 \sum_{l} u_{l} u_{l t}-c \sum_{i} F^{i i}|\nabla u|^{2} \\
& \geqslant \sum_{i, j, l} F^{i j}\left(w_{i j}\right)_{l} u_{l}-\sum_{i, l} F^{i i}\left(u_{i}^{2}-\frac{1}{2}|\nabla u|^{2}\right)_{l} u_{l}-2 \sum_{l} u_{l} u_{l t} \\
& \quad-c \sum_{i} F^{i i}|\nabla u|^{2}-\sum_{i, l} F^{i i} \nabla_{l}\left(S_{g_{0}}\right)_{i i} u_{l} \\
& \geqslant \sum_{l}\left(F_{l}-2 u_{t l}\right) u_{l}-c A_{0}{ }^{-1} \sum_{i} F^{i i}|\nabla u|^{4} \\
& \geqslant(\varepsilon-2) h^{\prime}\left(r_{\varepsilon}^{1 / 2} e^{(\varepsilon-2) u}\right) r_{\varepsilon}^{1 / 2} e^{(\varepsilon-2) u}|\nabla u|^{2}-c A_{0}{ }^{-1} \sum_{i} F^{i i}|\nabla u|^{4},
\end{aligned}
$$

where we have used (8) and (19). Here $c$ is a constant independent of $u$, but it may vary from line to line. The term $\sum_{i, j} F^{i j}\left(-2 \frac{\rho_{i} \rho_{j}}{\rho}+\rho_{i j}\right)|\nabla u|^{2}$ is bounded from below by $-10 b_{0}^{2}|\nabla u|^{2} \sum_{j} F^{j j}$. For the term $F^{i j} u_{i l} u_{j l}$ we have the following crucial lemma.

LEMMA 2 [18]. - There is a constant $A_{0}$ sufficient large (depending only on $n$, and $\left.\left\|g_{0}\right\|_{C^{3}\left(B_{1}\right)}\right)$, such that,

$$
\sum_{i, j, l} F^{i j} u_{i l} u_{j l} \geqslant A_{0}^{-3 / 4}|\nabla u|^{4} \sum_{i \geqslant 1} F^{i i} .
$$


Altogether gives us

$$
\left(A_{0}^{-3 / 4}-c A_{0}^{-1}\right) \rho|\nabla u|^{4} \sum_{i} F^{i i} \leqslant 10 b_{0}^{2}|\nabla u|^{2} \sum_{i} F^{i i}+c \rho\left(1+e^{(\varepsilon-2) u}\right)|\nabla u|^{2} .
$$

By the Newton-McLaurin inequality and the fact that $h^{\prime}(t) \geqslant 1$ for any $t \geqslant 0$, it is easy to check that

$$
\sum_{i} F^{i i} \geqslant 1
$$

which, together with (23), proves the local gradient estimate

$$
|\nabla u|^{2} \leqslant C\left(1+e^{(2-\varepsilon) \inf _{(x, t) \in B_{r} \times[0, T]} u(x, t)}\right),
$$

for some constant $C>0$ depending only on $\left(B_{r}, g_{0}\right)$.

Now we show the local estimates for second order derivatives. Since $e^{-2 u} g_{0} \in \Gamma_{2}^{+}$, to bound $\left|\nabla^{2} u\right|$ we only need bound $\Delta u$ from above. This is a well-known fact, see for instance [18]. Set

$$
G=\rho\left(\Delta u+|\nabla u|^{2}\right),
$$

where $\rho$ is defined as above. Let $\left(y_{0}, t_{0}\right)$ be a maximum point of $G$ in $M \times[0, T]$. Without loss of generality, we assume $G\left(y_{0}, t_{0}\right)>1+2 \max H(x, t), t_{0}>0$ and $\left(u_{i j}\right)$ is diagonal at $\left(y_{0}, t_{0}\right)$. Recall that $H=\rho|\nabla u|^{2}$. Hence we have

$$
0<\rho \Delta u\left(y_{0}, t_{0}\right) \leqslant G\left(y_{0}, t_{0}\right) \leqslant 2 \rho \Delta u\left(y_{0}, t_{0}\right) .
$$

At $\left(y_{0}, t_{0}\right)$, we have

$$
\begin{aligned}
& 0 \leqslant G_{t}=\rho \sum_{l}\left(u_{l l t}+2 u_{l} u_{l t}\right), \\
& 0=G_{j}=\frac{\rho_{j}}{\rho} G+\rho \sum_{l \geqslant 1}\left(u_{l l j}+2 u_{l} u_{l j}\right), \quad \text { for any } j, \\
& 0 \geqslant\left(G_{i j}=\frac{\rho \rho_{i j}-2 \rho_{i} \rho_{j}}{\rho^{2}} G+\rho \sum_{l \geqslant 1}\left(u_{l l i j}+2 u_{l i} u_{l j}+2 u_{l} u_{l i j}\right)\right) .
\end{aligned}
$$

Recall that $F^{i j}=\frac{\partial}{\partial w_{i j}} F$ is nonnegative definite. Hence, we have

$$
\begin{aligned}
0 \geqslant & \sum_{i, j \geqslant 1} F^{i j} G_{i j}-2 G_{t} \\
\geqslant & \sum_{i, j \geqslant 1} F^{i j} \frac{\rho \rho_{i j}-2 \rho_{i} \rho_{j}}{\rho^{2}} G+\rho \sum_{i, j, l \geqslant 1} F^{i j}\left(u_{i j l l}+2 u_{l i} u_{l j}+2 u_{l} u_{l i j}\right) \\
& -2 \rho \sum_{l}\left(u_{l l t}+2 u_{l} u_{l t}\right)-C \rho \sum_{i}\left(\left|u_{i i}\right|+\left|u_{i}\right|\right) \sum_{i, j}\left|F^{i j}\right|,
\end{aligned}
$$

where the last term comes from the commutators related to the curvature tensor of $g_{0}$ and its derivatives. From the definition of $\rho$, we have

$$
\sum_{i, j \geqslant 1} F^{i j} \frac{\rho \rho_{i j}-2 \rho_{i} \rho_{j}}{\rho^{2}} G \geqslant-C \sum_{i, j \geqslant 1}\left|F^{i j}\right| \frac{1}{\rho} G .
$$


By the concavity of $\sigma_{2}^{1 / 2}$, we have

$$
\begin{aligned}
\sum_{i, j, l \geqslant 1} F^{i j} u_{i j l l} & =\sum_{i, j, l \geqslant 1} F^{i j} w_{i j l l}-\sum_{i, j, l \geqslant 1} F^{i j}\left(u_{i} u_{j}-\frac{1}{2}|\nabla u|^{2}\left(g_{0}\right)_{i j}+\left(S_{g_{0}}\right)_{i j}\right)_{l l} \\
& \geqslant \sum_{l} F_{l l}-\sum_{i, j, l \geqslant 1} F^{i j}\left(u_{i} u_{j}-\frac{1}{2}|\nabla u|^{2}\left(g_{0}\right)_{i j}+\left(S_{g_{0}}\right)_{i j}\right)_{l l}
\end{aligned}
$$

We also have

$$
\begin{aligned}
\sum_{i, j, l} F^{i j} u_{l} u_{l i j}= & \sum_{i, j, l} F^{i j} u_{l}\left(w_{i j}\right)_{l}-\sum_{i, j, l} F^{i j} u_{l}\left(u_{i} u_{j}-\frac{1}{2}|\nabla u|^{2}\left(g_{0}\right)_{i j}+\left(S_{g_{0}}\right)_{i j}\right)_{l} \\
& +\sum_{i, j, l} F^{i j} u_{l}\left(u_{l i j}-u_{i j l}\right) \\
= & \sum_{l} F_{l} u_{l}-\sum F^{i j} u_{l}\left(u_{i} u_{j}-\frac{1}{2}|\nabla u|^{2}\left(g_{0}\right)_{i j}+\left(S_{g_{0}}\right)_{i j}\right)_{l} \\
& +\sum_{i, j, l} F^{i j} u_{l}\left(u_{l i j}-u_{i j l}\right) .
\end{aligned}
$$

Hence, we have

$$
\begin{aligned}
& \sum_{i, j, l \geqslant 1} F^{i j}\left(u_{i j l l}+2 u_{l i} u_{l j}+2 u_{l} u_{l i j}\right) \\
& \geqslant \sum_{l}\left(F_{l l}+2 F_{l} u_{l}\right)-2 \sum_{i, j, l} F^{i j} u_{i} u_{j l l}+\sum_{j, k, l} F^{j j} u_{k} u_{k l l} \\
& \quad-2 \sum_{i, j, l} F^{i j} u_{l}\left(u_{i} u_{j}-\frac{1}{2}|\nabla u|^{2}\left(g_{0}\right)_{i j}+\left(S_{g_{0}}\right)_{i j}\right)_{l} \\
& \quad+\sum_{i, k, l} F^{i i}\left(u_{k l}\right)^{2}-C\left(1+\frac{G}{\rho}\right) \sum_{i, j}\left|F^{i j}\right| .
\end{aligned}
$$

From (24) and Eq. (8), we have

$$
\rho \sum_{l}\left(F_{l l}+2 F_{l} u_{l}\right) \geqslant 2 \rho \sum_{l}\left(u_{t l l}+2 u_{l} u_{t l}\right)-C(2-\varepsilon) G\left(1+e^{(\varepsilon-2) u}\right) .
$$

The term $-2 \sum_{i, j, l} F^{i j} u_{i} u_{j l l}+\sum_{j, k, l} F^{j j} u_{k} u_{k l l}$ can be controlled as in [18] with the help of (25). And the other terms in (29) can easily be estimated. On the other hand, it follows from the positivity of $\left(F^{i j}\right)$ that

$$
\sum_{i, j}\left|F^{i j}\right| \leqslant C \sum_{i} F^{i i}
$$

This completes the proof of the theorem.

From the local estimates, we have

COROllary 1.- If "bubble" occurs, i.e., $\inf _{M \times\left[0, T^{*}\right)} u=-\infty$, then there is a positive constant $c_{0}>0$ such that

$$
\lim _{\delta \rightarrow 0} \lim _{t \rightarrow T^{*}} V_{\varepsilon}\left(g, B_{\delta}\right)>c_{0}
$$




\section{A Poincaré inequality and a Sobolev inequality}

The Sobolev inequality is a very important analytic tool in many problems arising from analysis and geometry. It plays a crucial role in the resolution of the Yamabe problem, which was solved completely by Yamabe [42], Trudinger [36], Aubin [2] and Schoen [32]. See various optimal Sobolev inequalities in [26]. In this section we are interested in a similar type inequality for the class of fully nonlinear conformal operators $\sigma_{k}\left(\nabla^{2} u+d u \otimes d u-\frac{|\nabla u|^{2}}{2} g_{0}+S_{g_{0}}\right)$. In [20], the Sobolev inequality was generalized to the operator $\sigma_{k}\left(\nabla^{2} u+d u \otimes d u-\frac{|\nabla u|^{2}}{2} g_{0}+S_{g_{0}}\right)$ for $k<n / 2$, if the underlying manifold is locally conformally flat. Namely,

THEOREM 4 [18]. - Let $\left(M^{n}, g_{0}\right)$ be a compact, oriented Riemannian manifold with $g_{0} \in \Gamma_{k}^{+}$ and $k<n / 2$. Assume that $\left(M, g_{0}\right)$ is locally conformally flat, then there exists a positive constant $C>0$ depending only on $n, k$ and $\left(M, g_{0}\right)$ such that for any $C^{2}$ function $u$ with $e^{-2 u} g_{0} \in \mathcal{C}_{k}\left(\left[g_{0}\right]\right)$ we have

$$
\int_{M} \sigma_{k}\left(e^{-2 u} g_{0}\right) d \operatorname{vol}\left(e^{-2 u} g_{0}\right) \geqslant C \operatorname{vol}\left(e^{-2 u} g_{0}\right)^{\frac{n-2 k}{n}} .
$$

Equivalently, for such a function $u$ we have

$$
\begin{aligned}
& \int_{M} e^{(2 k-n) u} \sigma_{k}\left(\nabla^{2} u+d u \otimes d u-\frac{|\nabla u|^{2}}{2} g_{0}+S_{g_{0}}\right) d \operatorname{vol}\left(g_{0}\right) \\
& \quad \geqslant C\left(\int_{M} e^{-n u} d \operatorname{vol}\left(g_{0}\right)\right)^{\frac{n-2 k}{n}} .
\end{aligned}
$$

When $k=1$, inequality (33) is just the Sobolev inequality. The proof of Theorem 4 uses a Yamabe type flow. See also the work of [13].

In this section, we establish the Sobolev inequality for $k=2$ without the flatness condition.

THEOREM 5. - Let $\left(M, g_{0}\right)$ be a compact, oriented Riemannian manifold with $g_{0} \in \Gamma_{2}^{+}$and the dimension $n>4$. Then there exists a positive constant $C>0$ depending only on $\left(M, g_{0}\right)$ such that for any $C^{2}$ function $u$ with $e^{-2 u} g_{0} \in \mathcal{C}_{2}\left(\left[g_{0}\right]\right)$ we have

$$
\int_{M} \sigma_{2}\left(e^{-2 u} g_{0}\right) d \operatorname{vol}\left(e^{-2 u} g_{0}\right) \geqslant C \operatorname{vol}\left(e^{-2 u} g_{0}\right)^{\frac{n-4}{n}} .
$$

Equivalently, for such a function $u$ we have

$$
\begin{gathered}
\int_{M} e^{(4-n) u} \sigma_{2}\left(\nabla^{2} u+d u \otimes d u-\frac{|\nabla u|^{2}}{2} g_{0}+S_{g_{0}}\right) d \operatorname{vol}\left(g_{0}\right) \\
\geqslant C\left(\int_{M} e^{-n u} d \operatorname{vol}\left(g_{0}\right)\right)^{\frac{n-4}{n}} .
\end{gathered}
$$

First we prove a Poincare type inequality, which will be used in the proof of our Sobolev inequality. The usual Poincaré type inequality is associated to the first eigenvalue problem. In our case, there is a nonlinear eigenvalue problem, which was studied in [21]. 
Proposition 5. - Let $\left(M, g_{0}\right)$ be a compact manifold with $g_{0} \in \Gamma_{k}^{+}$. Then there is a function $u$ with $e^{-2 u} g_{0} \in \Gamma_{k}^{+}$satisfying

$$
\sigma_{k}\left(\nabla^{2} u+d u \otimes d u-\frac{|\nabla u|^{2}}{2} g_{0}+S_{g_{0}}\right)=\lambda_{1}>0
$$

Moreover the constant $\lambda_{1}$ is unique and the solution is unique up to a constant.

An elliptic method was used in the proof, which was motivated by a method introduced in [30]. See also [41] for a Hessian operator. In view of Proposition 5, one may guess that

$$
\begin{aligned}
& \int e^{2 k u} \sigma_{k}\left(\nabla^{2} u+d u \otimes d u-\frac{|\nabla u|^{2}}{2} g_{0}+S_{g_{0}}\right) d \operatorname{vol}\left(e^{-2 u} g_{0}\right) \\
& \quad \geqslant \lambda_{1} \int e^{2 k u} d \operatorname{vol}\left(e^{-2 u} g_{0}\right),
\end{aligned}
$$

for any $u$ with $e^{-2 u} g_{0} \in \Gamma_{k}^{+}$. It is easy to see that when $k=1$ inequality (36) holds. In fact it is the Poincaré inequality. In this section, we show that (36) holds for $k=2$ by flow (8) with $\varepsilon=2$.

Proposition 6. - Let $\left(M, g_{0}\right)$ be a compact, oriented Riemannian manifold with $g_{0} \in \Gamma_{2}^{+}$ and the dimension $n>4$. Then for any $C^{2}$ function $u$ with $e^{-2 u} g_{0} \in \mathcal{C}_{2}\left(\left[g_{0}\right]\right)$ we have

$$
\int_{M} \sigma_{2}\left(e^{-2 u} g_{0}\right) d \operatorname{vol}\left(e^{-2 u} g_{0}\right) \geqslant \lambda_{1} \int e^{4 u} d \operatorname{vol}\left(e^{-2 u} g_{0}\right) .
$$

Equivalently, for such a function $u$ we have

$$
\begin{aligned}
& \int_{M} e^{(4-n) u} \sigma_{2}\left(\nabla^{2} u+d u \otimes d u-\frac{|\nabla u|^{2}}{2} g_{0}+S_{g_{0}}\right) d \operatorname{vol}\left(g_{0}\right) \\
& \quad \geqslant \lambda_{1} \int e^{(4-n) u} d \operatorname{vol}\left(g_{0}\right) .
\end{aligned}
$$

Proof. - To prove the proposition, we consider flow (8) with $\varepsilon=2$. We want to show that the flow converges globally to a solution obtained in Proposition 5. By Theorem 3, we have

$$
\left|\nabla^{2} u\right|+|\nabla u|^{2}(x, t) \leqslant C,
$$

where $C$ is a constant independent of $(x, t) \in M \times\left[0, T^{*}\right)$. Since the flow preserves the functional $V_{2}$, in view of (39) we have that $|u| \leqslant C$, for some constant $C>0$. Now following the method in [19] we can show that

$$
\sigma_{2}(g)>c_{0},
$$

for some constant $c_{0}$ independent of $t$. See the proof in the next section. Hence, this flow exists globally and is uniformly elliptic. By the result of Krylov, $g(t) \in C^{4+\alpha, 2+\alpha}$. Since the flow satisfies (9), one can show that for any sequence of $\left\{t_{i}\right\}$ with $t_{i} \rightarrow \infty$ there is a subsequence, still denoted by $\left\{t_{i}\right\}$, such that $g\left(t_{i}\right)$ converges strongly to $g^{*}$, which satisfies (35). On the other hand, $V_{2}\left(g^{*}\right) \equiv V_{2}(g(t))$. By the uniqueness in Proposition 5, one can show that the flow globally converges to $g^{*}$. Since the flow preserves $V_{2}$ and decreases $\mathcal{F}_{2}$, we have

$$
\mathcal{F}_{2}(g) \geqslant \mathcal{F}_{2}\left(g^{*}\right),
$$

$4^{e}$ SÉRIE - TOME $39-2006-\mathrm{N}^{\circ} 4$ 
for any $g \in \mathcal{C}_{2}$. This is the Poincaré inequality that we want to prove.

Proof of Theorem 5. - Let $g=e^{-2 u} g_{0}$. We have

$$
2 \sigma_{2}=\sum_{i, j} T^{i j} S_{i j}
$$

where $T(g)^{i j}=\sigma_{1}(g) g^{i j}-S(g)^{i j}$ is the so-called first Newton transformation. We will use the following formulas

$$
S(g)_{i j}=u_{i j}+u_{i} u_{j}-\frac{1}{2}|\nabla u|_{g_{0}}^{2}\left(g_{0}\right)_{i j}+S\left(g_{0}\right)_{i j}
$$

and

$$
\widetilde{\nabla}_{i j}^{2} u=u_{i j}+2 u_{i} u_{j}-|\nabla u|_{g_{0}}^{2}\left(g_{0}\right)_{i j},
$$

where $\widetilde{\nabla}$ are the derivatives w.r.t. $g$. Thus,

$$
\begin{aligned}
2 \sigma_{2}(g)= & \sum_{i, j} T(g)^{i j} \widetilde{\nabla}_{i j}^{2} u-\sum_{i, j} T(g)^{i j} u_{i} u_{j} \\
& +\frac{n-1}{2} \sigma_{1}(g)|\widetilde{\nabla} u|_{g}^{2}+\sum_{i, j} T(g)^{i j} S\left(g_{0}\right)_{i j} .
\end{aligned}
$$

Here we have used $\operatorname{tr} T(g)=(n-1) \sigma_{1}(g)$. Note that

$$
\sum_{i, j} T(g)^{i j} S\left(g_{0}\right)_{i j}>0
$$

thanks to Gårding's inequality

$$
\sum_{i, j} T(g)^{i j} S\left(g_{0}\right)_{i j} \geqslant 2 e^{2 u} \sigma_{2}^{1 / 2}(g) \sigma_{2}^{1 / 2}\left(g_{0}\right) .
$$

Due to an observation of Viaclovsky, $\sum_{i} \widetilde{\nabla}_{i} T(g)^{i j}=0$, we have

$$
\begin{aligned}
2 \int \sigma_{2}(g)= & -\int \sum_{i, j} T(g)^{i j} u_{i} u_{j} d \operatorname{vol}(g)+\frac{n-1}{2} \int \sigma_{1}(g)|\widetilde{\nabla} u|_{g}^{2} d \operatorname{vol}(g) \\
& +\int \sum_{i, j} T(g)^{i j} S\left(g_{0}\right)_{i j} d \operatorname{vol}(g) .
\end{aligned}
$$

Recall that $T(g)=\sigma_{1}(g) g-S(g)$. We have

$$
\begin{aligned}
& -\int \sum_{i, j} T(g)^{i j} u_{i} u_{j} d \operatorname{vol}(g) \\
& =-\int \sigma_{1}(g)|\widetilde{\nabla} u|_{g}^{2} d \operatorname{vol}(g)+\int \sum_{i, j} S(g)^{i j} u_{i} u_{j} d \operatorname{vol}(g) \\
& =-\int \sigma_{1}(g)|\widetilde{\nabla} u|_{g}^{2} d \operatorname{vol}(g)+\int \sum_{i, j} \widetilde{\nabla}^{i j} u u_{i} u_{j} d \operatorname{vol}(g)
\end{aligned}
$$




$$
-\frac{1}{2} \int|\widetilde{\nabla} u|_{g}^{4} d \operatorname{vol}(g)+\int \sum_{i, j} S\left(g_{0}\right)^{i j} u_{i} u_{j} d \operatorname{vol}(g)
$$

and

$$
\begin{aligned}
& \int \sum_{i, j} \widetilde{\nabla}^{i j} u u_{i} u_{j} d \operatorname{vol}(g) \\
& =\frac{1}{2} \int \sum_{i} \widetilde{\nabla}^{i}\left(|\widetilde{\nabla} u|_{g}^{2}\right) u_{i} d \operatorname{vol}(g)=-\frac{1}{2} \int|\widetilde{\nabla} u|_{g}^{2} \operatorname{tr}\left(\widetilde{\nabla}^{2} u\right) d \operatorname{vol}(g) \\
& =-\frac{1}{2} \int \sigma_{1}(g)|\widetilde{\nabla} u|_{g}^{2}+\frac{n-2}{4} \int|\widetilde{\nabla} u|_{g}^{4}+\int \frac{1}{2} \sigma_{1}\left(g_{0}\right)|\widetilde{\nabla} u|_{g}^{2} e^{2 u} d \operatorname{vol}(g) .
\end{aligned}
$$

Hence

$$
\begin{aligned}
(47)- & \int \sum_{i, j} T(g)^{i j} u_{i} u_{j} d \operatorname{vol}(g) \\
= & -\frac{3}{2} \int \sigma_{1}(g)|\widetilde{\nabla} u|_{g}^{2}+\frac{n-4}{4} \int|\widetilde{\nabla} u|_{g}^{4}+\int \sum_{i, j} S\left(g_{0}\right)^{i j} u_{i} u_{j}+\frac{1}{2} \int \sigma_{1}\left(g_{0}\right)|\widetilde{\nabla} u|_{g}^{2} e^{2 u},
\end{aligned}
$$

where all integrals are w.r.t. $g$. (44) and (47) give us

$$
\begin{aligned}
2 \int \sigma_{2}(g) d \operatorname{vol}(g) \\
=\frac{n-4}{2} \int \sigma_{1}(g)|\widetilde{\nabla} u|_{g}^{2} d \operatorname{vol} g+\frac{n-4}{4} \int|\widetilde{\nabla} u|_{g}^{4} d \operatorname{vol}(g) \\
\quad+\int \sum_{i, j} T^{i j} S\left(g_{0}\right)_{i j} d \operatorname{vol}(g)+\int \sum_{i, j} S\left(g_{0}\right)^{i j} u_{i} u_{j} d \operatorname{vol}(g) \\
\quad+\frac{1}{2} \int \sigma_{1}\left(g_{0}\right)|\widetilde{\nabla} u|_{g}^{2} e^{2 u} d \operatorname{vol}(g) .
\end{aligned}
$$

Finally, we obtain

$$
\begin{aligned}
2 \int \sigma_{2}(g) d \operatorname{vol}(g) \\
=\frac{n-4}{2} \int \sigma_{1}(g)|\nabla u|_{g_{0}}^{2} e^{2 u} d \operatorname{vol} g+\frac{n-4}{4} \int|\nabla u|_{g_{0}}^{4} e^{4 u} d \operatorname{vol}(g) \\
\quad+\int \sum_{i, j} T^{i j} S\left(g_{0}\right)_{i j} d \operatorname{vol}(g)+\int \sum_{i, j} S\left(g_{0}\right)^{i j} u_{i} u_{j} d \operatorname{vol}(g) \\
\quad+\frac{1}{2} \int \sigma_{1}\left(g_{0}\right)|\nabla u|_{g_{0}}^{2} e^{4 u} d \operatorname{vol}(g)
\end{aligned}
$$

Recall (43) and positivity of $\sigma_{1}(g)$ and $\sigma_{1}\left(g_{0}\right)$. Using the estimates

$$
\sum_{i, j} S\left(g_{0}\right)^{i j} u_{i} u_{j} \geqslant-c|\nabla u|_{g_{0}}^{2} e^{4 u} \geqslant-\frac{n-4}{8}|\nabla u|_{g_{0}}^{4} e^{4 u}-\frac{2 c^{2}}{n-4} e^{4 u}
$$

we deduce

$$
2 \int \sigma_{2}(g) d \operatorname{vol}(g) \geqslant \frac{n-4}{8} \int|\nabla u|_{g_{0}}^{4} e^{4 u} d \operatorname{vol}(g)-c \int e^{4 u} d \operatorname{vol}(g) .
$$


In view of the Poincaré inequality (37), the Sobolev inequality (33) follows from (51).

We remark that a similar method was used to obtain Sobolev inequalities on locally conformally flat manifolds by González in [13]. The argument given in the next section will provide another proof of Theorem 5.

\section{Global convergence of flow (8) when $\varepsilon>0$}

Proposition 7. - For any $\varepsilon \in(0,2]$, flow (8) converges globally to $u_{\varepsilon}$, which satisfies (6).

Proof. - For any $t \in\left[0, T^{*}\right)$, set

$$
m(t)=\min _{(x, s) \in M \times[0, t]} u(x, s) .
$$

If $\inf _{t \in\left[0, T^{*}\right)} m(t)>-\infty$, then by estimates given in Section 3, we have a uniform bound of $|\nabla u|^{2}+\left|\nabla^{2} u\right|$. Since flow (8) preserves the functional $V_{\varepsilon}$, we have a uniform $C^{2}$ bound. Now we claim that there is a constant $c>0$ such that

$$
F(x, t) \geqslant c>0, \quad \text { for any }(x, t) \in M \times\left[0, T^{*}\right) .
$$

Recall that $F=\sigma_{2}^{1 / 2}\left(\nabla^{2} u+d u \otimes d u-\frac{|\nabla u|^{2}}{2} g_{0}+S_{g_{0}}\right)$. We will prove the claim at the end of the proof. (52) implies that flow (8) is uniformly elliptic in $M \times\left[0, T^{*}\right)$. Hence, by Krylov's result, $u$ has a uniform bound for higher order derivatives, which implies first that $T^{*}=\infty$, the global existence. The global convergence of $(8)$ with $\varepsilon \in(0,2]$ follows now closely the argument presented in [19], which, in turn, follows closely the argument given in [35] and [1]. Therefore, to prove the proposition, we only need to exclude that

$$
\inf _{t \in\left[0, T^{*}\right)} m(t)=-\infty
$$

We assume by contradiction that $\inf _{t \in\left[0, T^{*}\right)} m(t)=-\infty$. Let $T_{i}$ be a sequence tending to $T^{*}$ with $m\left(T_{i}\right) \rightarrow-\infty$ as $i \rightarrow \infty$. Let $\left(x_{i}, t_{i}\right) \in M \times\left[0, T_{i}\right]$ with $u\left(x_{i}, t_{i}\right)=m\left(T_{i}\right)$. Fixing $\delta \in\left(\frac{2}{5}, \frac{1}{2}\right)$, we consider $r_{i}=\frac{\varepsilon}{2}\left|m\left(T_{i}\right)\right| e^{(1-\delta \varepsilon) m\left(T_{i}\right)}$. Clearly, we have $r_{i} \rightarrow 0$. It follows from Theorem 3 that for sufficiently large $i$

$$
\begin{aligned}
u\left(x, t_{i}\right) & \leqslant m\left(T_{i}\right)+|\nabla u| r_{i} \\
& \leqslant m\left(T_{i}\right)+C e^{\left(\frac{\varepsilon}{2}-1\right) m\left(T_{i}\right)} \frac{\varepsilon}{2}\left|m\left(T_{i}\right)\right| e^{(1-\delta \varepsilon) m\left(T_{i}\right)} \\
& =m\left(T_{i}\right)+C \frac{\varepsilon}{2}\left|m\left(T_{i}\right)\right| e^{\varepsilon(1 / 2-\delta) m\left(T_{i}\right)} \\
& \leqslant(1-\kappa) m\left(T_{i}\right), \quad \forall x \in B\left(x_{i}, r_{i}\right),
\end{aligned}
$$

for some $\kappa \in\left(0,\left(\delta-\frac{2}{n}\right) \varepsilon\right)$. Note that $\delta-\frac{2}{n}>0$, for $n \geqslant 5$. Therefore, we obtain

$$
\begin{aligned}
\int_{B\left(x_{i}, r_{i}\right)} e^{2 \varepsilon u} d \operatorname{vol}(g) & \geqslant \int_{B\left(x_{i}, r_{i}\right)} e^{(2 \varepsilon-n) m\left(T_{i}\right)(1-\kappa)} d \operatorname{vol}\left(g_{0}\right) \geqslant C e^{(2 \varepsilon-n) m\left(T_{i}\right)(1-\kappa)} r_{i}^{n} \\
& \geqslant C\left(\frac{\left|m\left(T_{i}\right)\right| \varepsilon}{2}\right)^{n} \rightarrow \infty .
\end{aligned}
$$

Hence, this fact contradicts the boundedness of $V_{\varepsilon}$. 
Now it remains to prove claim (52). For any $0<T<T^{*}$, set

$$
T_{1}:=\inf \left\{T^{\prime} \in[0, T] \mid \forall(x, t) \in M \times\left[T^{\prime}, T\right], r_{\varepsilon}^{1 / 2}(g(t)) e^{(\varepsilon-2) u(x, t)}<1 / 2\right\} .
$$

It is clear that $\forall t \in\left[0, T_{1}\right]$ we have $r_{\varepsilon}(g(t))>C$ for positive constant $C>0$ independent of $T$. Let us consider a function $H: M \times\left[0, T_{1}\right]$ defined by

$$
\begin{aligned}
H & :=\frac{1}{2}\left(h\left(\sigma_{2}^{1 / 2}\left(\nabla^{2} u+d u \otimes d u-\frac{|\nabla u|^{2}}{2} g_{0}+S_{g_{0}}\right)-h\left(r_{\varepsilon}^{1 / 2}(g) e^{(\varepsilon-2) u}\right)\right)-e^{-u}\right. \\
& =u_{t}+\frac{1}{2} s_{\varepsilon}(g)-e^{-u} .
\end{aligned}
$$

We first compute the evolution equation for $\sigma_{2}$. A direct computation, see for instance Lemma 2 in [19], gives

$$
\begin{aligned}
\frac{d}{d t} \sigma_{2} & =2 \sigma_{2} g \cdot \frac{d}{d t}\left(g^{-1}\right)+\operatorname{tr}\left\{T_{1}\left(S_{g}\right) g^{-1} \frac{d}{d t} S_{g}\right\} \\
& =4 \sigma_{2}(g) u_{t}+\operatorname{tr}\left\{T_{1}\left(S_{g}\right) g^{-1} \widetilde{\nabla}_{g}^{2}\left(u_{t}\right)\right\}
\end{aligned}
$$

where $\widetilde{\nabla}$ is the derivatives with respect to the evolved metric $g$. Without loss of generality, we assume that the minimum of $H$ is achieved at $\left(x_{0}, t_{0}\right) \in M \times\left(0, T_{1}\right]$. We will show that there is a constant $c_{0}>0$ independent of $T$ such that

$$
\sigma_{2}(g)\left(x_{0}, t_{0}\right)>c_{0}
$$

Since $|u|$ has a uniform bound, without loss of generality we may assume that at $\left(x_{0}, t_{0}\right)$

$$
e^{-2 u} \sigma_{2}^{1 / 2}(g)<1 / 2
$$

Recall that $h(t)=2 \log t$ for $t<1$. Hence, in a small neighborhood of $\left(x_{0}, t_{0}\right)$

$$
H=\log \left(e^{-2 u} \sigma_{2}^{1 / 2}(g)\right)-\frac{1}{2} h\left(r_{\varepsilon}^{1 / 2}(g) e^{(\varepsilon-2) u}\right)-e^{-u} .
$$

Let us use $O(1)$ to denote terms with a uniform bound. We have near $\left(x_{0}, t_{0}\right)$

$$
\begin{aligned}
\frac{d}{d t} H= & \frac{1}{2 \sigma_{2}(g)} \operatorname{tr}\left\{T_{1}\left(S_{g}\right) g^{-1} \widetilde{\nabla}_{g}^{2}\left(u_{t}\right)\right\} \\
& -\frac{1}{4} h^{\prime}\left(r_{\varepsilon}^{1 / 2}(g) e^{(\varepsilon-2) u}\right) \frac{d r_{\varepsilon}(g)}{d t} r_{\varepsilon}^{-1 / 2}(g) e^{(\varepsilon-2) u} \\
& +\left[e^{-u}+\frac{2-\varepsilon}{2} h^{\prime}\left(r_{\varepsilon}^{1 / 2}(g) e^{(\varepsilon-2) u}\right) r_{\varepsilon}^{1 / 2}(g) e^{(\varepsilon-2) u}\right] u_{t} \\
\geqslant & \frac{1}{2 \sigma_{2}(g)} \operatorname{tr}\left\{T_{1}\left(S_{g}\right) g^{-1} \widetilde{\nabla}_{g}^{2}(H)\right\}+\frac{1}{2 \sigma_{2}(g)} \operatorname{tr}\left\{T_{1}\left(S_{g}\right) g^{-1} \widetilde{\nabla}_{g}^{2}\left(e^{-u}\right)\right\} \\
& +\left[e^{-u}+\frac{2-\varepsilon}{2} h^{\prime}\left(r_{\varepsilon}^{1 / 2}(g) e^{(\varepsilon-2) u}\right) r_{\varepsilon}^{1 / 2}(g) e^{(\varepsilon-2) u}\right] u_{t},
\end{aligned}
$$

$4^{e}$ SÉRIE - TOME $39-2006-\mathrm{N}^{\circ} 4$ 
where we have used $\frac{d r_{\varepsilon}(g)}{d t} \leqslant 0$. See Lemma 1. Let $F=\log \sigma_{2}\left(\nabla^{2} u+d u \otimes d u-\frac{|\nabla u|^{2}}{2} g_{0}+S_{g_{0}}\right)$ and $F^{i j}=\frac{\partial}{\partial w_{i j}} F$. Since $\left(x_{0}, t_{0}\right)$ is the minimum of $H$ in $M \times\left[0, T_{1}\right]$, at this point, we have

$$
\begin{gathered}
\frac{d H}{d t} \leqslant 0 \\
0=H_{l}=\frac{1}{2} \sum_{i j} F^{i j} w_{i j l}+\left(e^{-u}+\frac{2-\varepsilon}{2} h^{\prime}\left(r_{\varepsilon}^{1 / 2}(g) e^{(\varepsilon-2) u}\right) r_{\varepsilon}^{1 / 2}(g) e^{(\varepsilon-2) u}\right) u_{l}=0 \quad \forall l
\end{gathered}
$$

and

$$
\left(H_{i j}\right) \quad \text { is nonnegative definite. }
$$

Note that

$$
\left(\widetilde{\nabla}_{g}^{2}\right)_{i j} H=H_{i j}+u_{i} H_{j}+u_{j} H_{i}-\sum_{l} u_{l} H_{l} \delta_{i j}=H_{i j},
$$

at $\left(x_{0}, t_{0}\right)$, where $H_{j}$ and $H_{i j}$ are the first and second derivatives with respect to the background metric $g_{0}$. We write $k(x, t):=\left(e^{-u}+\frac{2-\varepsilon}{2} h^{\prime}\left(r_{\varepsilon}^{1 / 2}(g) e^{(\varepsilon-2) u}\right) r_{\varepsilon}^{1 / 2}(g) e^{(\varepsilon-2) u}\right)$. We can check that $\forall(x, t) \in M \times\left[0, T_{1}\right]$ we have $C_{1}>k(x, t)>C_{2}$. Here the positive constants $C_{1}$ and $C_{2}$ are independent of $T$.

From the positivity of $\left(F^{i j}\right)$ and (54), we have

$$
\begin{aligned}
0 \geqslant & H_{t}-\frac{1}{2} \sum_{i, j} F^{i j} H_{i j} \\
\geqslant & \frac{1}{2 \sigma_{2}(g)} \operatorname{tr}\left\{T_{1}\left(S_{g}\right) \widetilde{\nabla}_{g}^{2}\left(e^{-u}\right)\right\}+k(x, t) u_{t} \\
= & \frac{1}{2} \sum_{i, j} F^{i j}\left\{\left(e^{-u}\right)_{i j}+u_{i}\left(e^{-u}\right)_{j}+u_{j}\left(e^{-u}\right)_{i}-u_{l}\left(e^{-u}\right)_{l} \delta_{i j}\right\}+k(x, t) u_{t} \\
= & \frac{1}{2} e^{-u} \sum_{i, j} F^{i j}\left\{-u_{i j}-u_{i} u_{j}+|\nabla u|^{2} \delta_{i j}\right\}+k(x, t) u_{t} \\
= & \frac{1}{2} e^{-u} \sum_{i, j} F^{i j}\left\{-w_{i j}+S\left(g_{0}\right)_{i j}+\frac{1}{2}|\nabla u|^{2} \delta_{i j}\right\}+k(x, t) u_{t} \\
\geqslant & \frac{1}{2} e^{-u} \sum_{i, j} F^{i j}\left\{-w_{i j}+S\left(g_{0}\right)_{i j}\right\}+k(x, t) u_{t} \\
= & \frac{1}{2} e^{-u} \sum_{i, j} F^{i j} S\left(g_{0}\right)_{i j}+O(1) \log \sigma_{2}(g)+O(1) \\
& -\frac{1}{2} k(x, t)\left(h\left(r_{\varepsilon}^{1 / 2}(g) e^{(\varepsilon-2) u}\right)+s_{\varepsilon}(g)\right) .
\end{aligned}
$$

Here we have used $\sum_{i, j} F^{i j} w_{i j}=\sum_{i, j} \frac{1}{\sigma_{2}(g)} \frac{\partial \sigma_{2}(g)}{\partial w_{i j}} w_{i j}=2$. Since $g_{0} \in \Gamma_{2}^{+}$, by Gårding's inequality [11],

$$
\sum_{i, j} F^{i j} S\left(g_{0}\right)_{i j}=\sum_{i, j} \frac{1}{\sigma_{2}(g)} \frac{\partial \sigma_{2}(g)}{w_{i j}} S\left(g_{0}\right)_{i j} \geqslant 2 e^{2 u} \frac{\sigma_{2}^{1 / 2}\left(g_{0}\right)}{\sigma_{2}^{1 / 2}(g)} .
$$

ANNALES SCIENTIFIQUES DE L'ÉCOLE NORMALE SUPÉRIEURE 
On the other hand, one can check $h\left(r_{\varepsilon}^{1 / 2}(g) e^{(\varepsilon-2) u}\right)+s_{\varepsilon}(g)$ is bounded from above, for $\|u\|_{C^{2}}$ is uniformly bounded. Now from (56) and (57), we have

$$
\begin{aligned}
0 & \geqslant e^{u} \frac{\sigma_{2}^{1 / 2}\left(g_{0}\right)}{\sigma_{2}^{1 / 2}(g)}+O(1) \log \sigma_{2}(g)+O(1) \\
& \geqslant \frac{c_{1}}{\sigma_{2}^{1 / 2}(g)}+c_{2} \log \sigma_{2}(g)-c_{3}
\end{aligned}
$$

for positive constants $c_{1}, c_{2}$ and $c_{3}$ independent of $T$. Clearly, this inequality implies that there is a constant $c_{0}>0$ independent of $T$ such that (54) holds. Namely

$$
\sigma_{2}(g) \geqslant c_{0}
$$

at point $\left(x_{0}, t_{0}\right)$. Hence, we have for any point $(x, t) \in M \times\left[0, T_{1}\right]$

$$
\begin{aligned}
\log & \left(e^{-2 u(x, t)} \sigma_{2}^{1 / 2}(g)(x, t)\right)-\frac{1}{2} h\left(r_{\varepsilon}^{1 / 2}(g) e^{(\varepsilon-2) u(x, t)}\right)-e^{-u(x, t)} \\
& =H(x, t) \\
& \geqslant H\left(x_{0}, t_{0}\right) \\
& =\log \left(e^{-2 u\left(x_{0}, t_{0}\right)} \sigma_{2}^{1 / 2}(g)\left(x_{0}, t_{0}\right)\right)-\frac{1}{2} h\left(r_{\varepsilon}^{1 / 2}(g) e^{(\varepsilon-2) u\left(x_{0}, t_{0}\right)}\right)-e^{-u\left(x_{0}, t_{0}\right)} \\
& \geqslant \log C_{1}-e^{C},
\end{aligned}
$$

provided $e^{-2 u(x, t)} \sigma_{2}^{1 / 2}(g)(x, t)<1$. It follows that $\sigma_{2}(g)(x, t) \geqslant C_{2}>0$ for some positive constant independent of $T$.

On $M \times\left[T_{1}, T\right]$, we consider a function $H: M \times\left[T_{1}, T\right]$ defined by $H=$ $\log \left(e^{-\varepsilon u} \sigma_{2}^{1 / 2}(g)\right)-e^{-u}$. By the same argument, there is a constant $c>0$ independent of $T$ such that $F(x, t) \geqslant c>0$ for any $(x, t) \in M \times\left[T_{1}, T\right]$. Hence, we deduce the claim (52). This finishes the proof of the proposition.

We remark that the Sobolev inequality, Theorem 2, implies that $r_{\varepsilon}$ has a positive lower bound. In the proof of claim (52), we avoided to use the Sobolev inequality.

Proof of Proposition 1. - By local estimates established in [18] (in fact similar local estimates as in Theorem 3 hold), we can use the argument given in the proof of Proposition 7 to show that the set of solutions of (6) with the bounded $\mathcal{F}_{2}$ and $V_{\varepsilon}\left(e^{-2 u} g_{0}\right)=1$ is compact for $\varepsilon \in(0,2]$. Hence, Proposition 7 implies that $Y_{\varepsilon}$ is achieved by a function $u_{\varepsilon}$, which clearly is a solution of (6). We may assume that $u_{\varepsilon}$ satisfies $V_{\varepsilon}\left(e^{-2 u_{\varepsilon}} g_{0}\right)=1$ and

$$
\sigma_{2}\left(\nabla^{2} u+d u \otimes d u-\frac{|\nabla u|^{2}}{2} g_{0}+S_{g_{0}}\right)=c e^{2(\varepsilon-2) u}
$$

where $c=Y_{\varepsilon}$. For any fixed metric $g$, the function $\widetilde{\mathcal{F}}_{2, \varepsilon}(g)$ is continuous on $\varepsilon$ so that $Y_{\varepsilon}$ is semicontinuous from above on $\varepsilon$. On the other hand, it follows from the Hölder's inequality, $Y_{\varepsilon}$ is semi-continuous from below on $\varepsilon$. Hence, $Y_{\varepsilon}$ is continuous and we have

$$
\lim _{\varepsilon \rightarrow 0} Y_{\varepsilon}=Y_{2}\left(M,\left[g_{0}\right]\right)<Y_{2}\left(\mathbb{S}^{n}\right) .
$$

If inf $u_{\varepsilon}$ has a uniform lower bound, then the estimates established in [18] imply that $\left\|u_{\varepsilon}\right\|_{C^{2}}$ is uniformly bounded. By the result of Evans-Krylov, $\left\|u_{\varepsilon}\right\|_{C^{2, \alpha}}$ is uniformly bounded for any 
$\alpha \in(0,1)$. Hence $u_{\varepsilon}$, by taking a subsequence, converges strongly in $C^{2, \alpha}$ to $u_{0}$, which is a solution of (1). Moreover, $u_{0}$ is a minimizer. Now suppose $\underline{\lim }_{\varepsilon \rightarrow 0}$ inf $u_{\varepsilon}=-\infty$. Let $\left(x_{\varepsilon}\right) \in M$ such that $u_{\varepsilon}\left(x_{\varepsilon}\right)=\min _{x \in M} u_{\varepsilon}(x)$. We consider a new function

$$
v_{\varepsilon}(y)=u\left(\exp _{x_{\varepsilon}} \delta_{\varepsilon} y\right)-u_{\varepsilon}\left(x_{\varepsilon}\right)
$$

and defined on $B_{\delta_{\varepsilon}^{-1}}$ with a pull-back metric $g_{\varepsilon}:=\left(\exp _{x_{\varepsilon}} \delta_{\varepsilon} \cdot\right)^{*} g_{0}$, where $\delta_{\varepsilon}=e^{\left(1-\frac{\varepsilon}{2}\right) u_{\varepsilon}\left(x_{\varepsilon}\right)}$. Since $u_{\varepsilon}\left(x_{\varepsilon}\right) \rightarrow-\infty, \delta_{\varepsilon} \rightarrow 0$ as $\varepsilon \rightarrow 0$. And one can check that $B_{\delta_{\varepsilon}^{-1}}$ tends to $\mathbb{R}^{n}$ and $g_{\varepsilon}$ to the standard Euclidean metric in any compact set in $\mathbb{R}^{n}$ for any $C^{k}$ norm. We can check that $v_{\varepsilon}$ satisfies the same Eq. (58) on $B_{\delta_{\varepsilon}^{-1}}$ with $S_{g_{0}}$ replaced by $S_{g_{\varepsilon}}$. By the local estimates in [18], $\left(v_{\varepsilon}\right)$ is uniformly bounded in $C^{2}$ on any fixed compact set. From the result of Evans-Krylov, it follows that $\left(v_{\varepsilon}\right)$ is uniformly bounded in $C^{2, \alpha}$ on any fixed compact set. Hence, $v_{\varepsilon}$ converges in any compact domain of $\mathbb{R}^{n}$ to an entire solution $u$ of the following equation on $\mathbb{R}^{n}$

$$
\sigma_{2}\left(\nabla^{2} u+d u \otimes d u-\frac{1}{2}|\nabla u|^{2} g_{\mathbb{R}^{n}}\right)=c_{0} e^{-4 u},
$$

with $c_{0}=Y_{2}\left(M,\left[g_{0}\right]\right)$. It is easy to check that $\int_{\mathbb{R}^{n}} e^{-n u} d \operatorname{vol}\left(g_{\mathbb{R}^{n}}\right) \leqslant 1$. In fact, we have

$$
\begin{aligned}
& \int_{B_{\delta_{\varepsilon}^{-1}}} e^{(2 \varepsilon-n) v_{\varepsilon}} d \operatorname{vol}\left(g_{\varepsilon}\right) \\
& =\delta_{\varepsilon}^{-n} e^{(n-2 \varepsilon) u_{\varepsilon}\left(x_{\varepsilon}\right)} \int_{B\left(x_{\varepsilon}, 1\right)} e^{(2 \varepsilon-n) u_{\varepsilon}} d \operatorname{vol}\left(g_{0}\right) \\
& =e^{(n / 2-2) \varepsilon u_{\varepsilon}\left(x_{\varepsilon}\right)} \int_{B\left(x_{\varepsilon}, 1\right)} e^{(2 \varepsilon-n) u_{\varepsilon}} d \operatorname{vol}\left(g_{0}\right) \leqslant V_{\varepsilon}\left(e^{-2 u_{\varepsilon}} g_{0}\right)=1 .
\end{aligned}
$$

Letting $\varepsilon \rightarrow 0$, the claim yields. By the classification of (59) given in [29] or [8], we have $c_{0} \geqslant Y_{2}\left(\mathbb{S}^{n}\right)>Y_{2}\left(M,\left[g_{0}\right]\right)$, which contradicts $c_{0}=Y_{2}\left(M,\left[g_{0}\right]\right)$.

\section{Existence}

In this section, we will construct a conformal metric $\tilde{g}$ such that $\widetilde{\mathcal{F}}_{2}(\tilde{g})<Y_{2}\left(\mathbb{S}^{n}\right)$ and $\tilde{g} \in \Gamma_{2}^{+}$. Our construction is inspired from Aubin's work [2]. See also [32]. The basic idea is to construct a suitable test function. But the more delicate point in our case, as already mentioned in the Introduction, is to keep the conformal metric in the admissible class $\Gamma_{2}^{+}$as in [20]. Fix a point $P \in M$. Assume $n \geqslant 5$. It follows from the work by Lee-Parker that there exists a conformal metric $g_{1}$ on $\mathcal{M}$ such that in a normal coordinate system for $g_{1}$ at $P$

$$
\begin{gathered}
R=O\left(r^{2}\right), \\
\Delta R=-\frac{1}{6}|W(P)|^{2}, \\
\operatorname{Ric}(P)=0, \\
\sqrt{\operatorname{det} g_{1}}=1+O\left(r^{5}\right),
\end{gathered}
$$

where $r=|x|$. We denote

$$
g_{v}=v^{-2} g_{1},
$$


where

$$
v(x)= \begin{cases}\lambda+r^{2}, & \text { if } x \in B\left(0, r_{0}\right), \\ \lambda+r_{0}^{2}, & \text { else. }\end{cases}
$$

We first need some estimates.

Lemma 3. - Assume

$$
A=g_{1}{ }^{-1}\left(\frac{\nabla_{g_{1}}^{2} v}{v}-\frac{1}{2} \frac{\left|\nabla_{g_{1}} v\right|^{2}}{v^{2}} g_{1}+S_{g_{1}}\right)
$$

where

$$
S_{g_{1}}=\frac{1}{n-2}\left(\operatorname{Ric}_{g_{1}}-\frac{R}{2(n-1)} g_{1}\right)
$$

Then we have

$$
\operatorname{tr}(A)=\frac{2 n \lambda}{\left(\lambda+r^{2}\right)^{2}}+\frac{O\left(r^{5}\right)}{\lambda+r^{2}}+\frac{R}{2(n-1)}
$$

and

$$
\operatorname{tr}\left(A^{2}\right)=\frac{4 n \lambda^{2}}{\left(\lambda+r^{2}\right)^{4}}+\frac{2 R \lambda}{(n-1)\left(\lambda+r^{2}\right)^{2}}-\frac{\operatorname{Ric}\left(\nabla_{g_{1}} v, \nabla_{g_{1}} v\right)}{\left(\lambda+r^{2}\right)^{2}}+O(r) .
$$

Proof. - By definition, we have

$$
\sigma_{2}\left(g_{v}\right)=v^{4} \sigma_{2}\left(g_{1}{ }^{-1}\left(\frac{\nabla_{g_{1}}^{2} v}{v}-\frac{1}{2} \frac{\left|\nabla_{g_{1}} v\right|^{2}}{v^{2}} g_{1}+S_{g_{1}}\right)\right) .
$$

It is clear that

$$
\begin{aligned}
\operatorname{tr}(A) & =\frac{\Delta_{g_{1}} v}{v}-\frac{n}{2} \frac{\left|\nabla_{g_{1}} v\right|^{2}}{v^{2}}+\operatorname{tr}\left(g_{1}^{-1} S_{g_{1}}\right) \\
& =\frac{\Delta_{g_{1}} v}{v}-\frac{n}{2} \frac{\left|\nabla_{g_{1}} v\right|^{2}}{v^{2}}+\frac{R}{2(n-1)},
\end{aligned}
$$

where

$$
\Delta_{g_{1}}=\frac{1}{\sqrt{\operatorname{det} g_{1}}} \sum_{i, j} \frac{\partial}{\partial x^{i}}\left(\sqrt{\operatorname{det} g_{1}} g_{1}^{i j} \frac{\partial}{\partial x^{j}}\right) .
$$

Recall

$$
\left|\nabla_{g_{1}} v\right|^{2}=4 r^{2}
$$

In view of (63), we have

$$
\Delta_{g_{1}} v=2 n+O\left(r^{5}\right)
$$

Therefore,

$$
\begin{aligned}
\operatorname{tr}(A) & =\frac{2 n+O\left(r^{5}\right)}{\lambda+r^{2}}-\frac{n}{2} \frac{4 r^{2}}{\left(\lambda+r^{2}\right)^{2}}+\frac{R}{2(n-1)} \\
& =\frac{2 n \lambda}{\left(\lambda+r^{2}\right)^{2}}+\frac{O\left(r^{5}\right)}{\lambda+r^{2}}+\frac{R}{2(n-1)} .
\end{aligned}
$$

$4^{e}$ SÉRIE - TOME 39 - 2006 - N ${ }^{\circ} 4$ 
It is also easy to check that

$$
\begin{aligned}
\operatorname{tr}\left(A^{2}\right)= & \frac{\left|\nabla_{g_{1}}^{2} v\right|^{2}}{v^{2}}+\frac{n\left|\nabla_{g_{1}} v\right|^{4}}{4 v^{4}}+\operatorname{tr}\left(\left(g_{1}{ }^{-1} S_{g_{1}}\right)^{2}\right) \\
& -\frac{\left|\nabla_{g_{1}} v\right|^{2} \Delta_{g_{1}} v}{v^{3}}+2 \operatorname{tr}\left(\frac{g_{1}{ }^{-1} \nabla_{g_{1}}^{2} v g_{1}{ }^{-1} S_{g_{1}}}{v}\right)-\frac{\left|\nabla_{g_{1}} v\right|^{2}}{v^{2}} \operatorname{tr}\left(g_{1}{ }^{-1} S_{g_{1}}\right) .
\end{aligned}
$$

A simple calculation gives us

$$
\begin{aligned}
\frac{n\left|\nabla_{g_{1}} v\right|^{4}}{4 v^{4}} & =\frac{4 n r^{4}}{\left(\lambda+r^{2}\right)^{4}}, \\
\operatorname{tr}\left(\left(g_{1}{ }^{-1} S_{g_{1}}\right)^{2}\right) & =O\left(r^{2}\right), \\
-\frac{\left|\nabla_{g_{1}} v\right|^{2} \Delta_{g_{1}} v}{v^{3}} & =-\frac{8 n r^{2}}{\left(\lambda+r^{2}\right)^{3}}+O(r), \\
-\frac{\left|\nabla_{g_{1}} v\right|^{2}}{v^{2}} \operatorname{tr}\left(g_{1}{ }^{-1} S_{g_{1}}\right) & =-\frac{2 r^{2} R}{(n-1)\left(\lambda+r^{2}\right)^{2}}, \\
g_{1}{ }^{-1} \nabla_{g_{1}}^{2} v & =2 I+O\left(r^{2}\right), \\
\operatorname{tr}\left(g_{1}{ }^{-1} \nabla_{g_{1}}^{2} v g_{1}{ }^{-1} S_{g_{1}}\right) & =2 \operatorname{tr}\left(g_{1}{ }^{-1} S_{g_{1}}\right)+O\left(r^{3}\right)=\frac{R}{n-1}+O\left(r^{3}\right), \\
2 \operatorname{tr}\left(\frac{g_{1}{ }^{-1} \nabla_{g_{1}}^{2} v g_{1}{ }^{-1} S_{g_{1}}}{v}\right) & =\frac{2 R}{(n-1)\left(\lambda+r^{2}\right)}+O(r) .
\end{aligned}
$$

To handle $\frac{\left|\nabla_{g_{1}}^{2} v\right|^{2}}{v^{2}}$, we recall the Bochner's formula

$$
\langle\nabla(\Delta v), \nabla v\rangle=-\left|\nabla_{g_{1}}^{2} v\right|^{2}+\frac{1}{2} \Delta\left(\left|\nabla_{g_{1}} v\right|^{2}\right)-\operatorname{Ric}\left(\nabla_{g_{1}} v, \nabla_{g_{1}} v\right) .
$$

Hence, we have

$$
\begin{aligned}
\left|\nabla_{g_{1}}^{2} v\right|^{2} & =-\langle\nabla(\Delta v), \nabla v\rangle+\frac{1}{2} \Delta\left(\left|\nabla_{g_{1}} v\right|^{2}\right)-\operatorname{Ric}\left(\nabla_{g_{1}} v, \nabla_{g_{1}} v\right) \\
& =-\left\langle\nabla\left(2 n+O\left(r^{5}\right)\right), \nabla v\right\rangle+\frac{1}{2} \Delta\left(4 r^{2}\right)-\operatorname{Ric}\left(\nabla_{g_{1}} v, \nabla_{g_{1}} v\right) \\
& =4 n-\operatorname{Ric}\left(\nabla_{g_{1}} v, \nabla_{g_{1}} v\right)+O\left(r^{5}\right) .
\end{aligned}
$$

From (70), (76) and (78), we have

$$
\begin{aligned}
\operatorname{tr}\left(A^{2}\right)= & \frac{4 n}{\left(\lambda+r^{2}\right)^{2}}+\frac{4 n r^{4}}{\left(\lambda+r^{2}\right)^{4}}-\frac{8 n r^{2}}{\left(\lambda+r^{2}\right)^{3}}+\frac{2 R}{(n-1)\left(\lambda+r^{2}\right)} \\
& -\frac{2 R r^{2}}{(n-1)\left(\lambda+r^{2}\right)^{2}}-\frac{\operatorname{Ric}\left(\nabla_{g_{1}} v, \nabla_{g_{1}} v\right)}{\left(\lambda+r^{2}\right)^{2}}+O(r) \\
= & \frac{4 n \lambda^{2}}{\left(\lambda+r^{2}\right)^{4}}+\frac{2 R \lambda}{(n-1)\left(\lambda+r^{2}\right)^{2}}-\frac{\operatorname{Ric}\left(\nabla_{g_{1}} v, \nabla_{g_{1}} v\right)}{\left(\lambda+r^{2}\right)^{2}}+O(r) .
\end{aligned}
$$

Lemma 4. - Assume $\beta \in\left(\frac{1}{2}, \frac{1}{4}\right)$. We have $\sigma_{1}\left(g_{v}\right)>0$ and $\sigma_{2}\left(g_{v}\right)>0$ in $B\left(0, \lambda^{\beta}\right)$. Moreover, if $n \geqslant 9$, we have

$$
\int_{B\left(0, \lambda^{\beta}\right)} \sigma_{2}\left(g_{v}\right) d \operatorname{vol}\left(g_{v}\right)=\lambda^{-\frac{n}{2}+2}\left\{2 n(n-1) B+C \Delta R(0) \lambda^{2}\right.
$$




$$
\left.+O\left(\lambda^{5 / 2}+\lambda^{n(1 / 2-\beta)}+\lambda^{2+(n-8)(1 / 2-\beta)}\right)\right\}
$$

and

$$
\int_{B\left(0, \lambda^{\beta}\right)} d \operatorname{vol}\left(g_{v}\right)=\lambda^{-\frac{n}{2}}\left[B+O\left(\lambda^{5 / 2}+\lambda^{n(1 / 2-\beta)}\right)\right],
$$

where the constants $B, C$ are given by

$$
\begin{gathered}
B=\int_{\mathbb{R}^{n}} \frac{1}{\left(1+|x|^{2}\right)^{n}} d x, \\
C=\int_{\mathbb{R}^{n}}\left(\frac{|x|^{2}}{2 n\left(1+|x|^{2}\right)^{n-2}}+\frac{2|x|^{4}}{n(n+2)\left(1+|x|^{2}\right)^{n-2}}\right) d x>0 .
\end{gathered}
$$

Proof. - It follows directly from (65) and (66)

$$
\begin{gathered}
\sigma_{1}\left(g_{v}\right)=v^{2}\left(\frac{2 n \lambda}{\left(\lambda+r^{2}\right)^{2}}+\frac{R}{2(n-1)}+O\left(r^{3}\right)\right), \\
\sigma_{2}\left(g_{v}\right)=\frac{v^{4}}{2}\left[\frac{4 n(n-1) \lambda^{2}}{\left(\lambda+r^{2}\right)^{4}}+\frac{2 \lambda R}{\left(\lambda+r^{2}\right)^{2}}+\frac{\operatorname{Ric}\left(\nabla_{g_{1}} v, \nabla_{g_{1}} v\right)}{\left(\lambda+r^{2}\right)^{2}}+O(r)\right] .
\end{gathered}
$$

Thus, the first part of the lemma is clear. On the other hand, we obtain

$$
\begin{aligned}
& \int_{B\left(0, \lambda^{\beta}\right)} \sigma_{2}\left(g_{v}\right) d \operatorname{vol}\left(g_{v}\right) \\
& =\int_{B\left(0, \lambda^{\beta}\right)} \frac{1}{\left(\lambda+r^{2}\right)^{n}}\left\{2 n(n-1) \lambda^{2}+R \lambda\left(\lambda+r^{2}\right)^{2}\right. \\
& \left.\quad+\frac{1}{2} \operatorname{Ric}\left(\nabla_{g_{1}} v, \nabla_{g_{1}} v\right)\left(\lambda+r^{2}\right)^{2}+O\left(r\left(\lambda+r^{2}\right)^{4}\right)\right\}\left(1+O\left(r^{5}\right)\right) d x .
\end{aligned}
$$

We can calculate

$$
\begin{aligned}
\operatorname{Ric}\left(\nabla_{g_{1}} v, \nabla_{g_{1}} v\right) & =4 \sum_{i, j} R_{i j}(x) x^{i} x^{j} \\
& =4 \sum_{i, j}\left(R_{i j}(0)+\sum_{k} R_{i j, k}(0) x^{k}+\sum_{k, l} \frac{1}{2} R_{i j, k l}(0) x^{k} x^{l}\right) x^{i} x^{j}+O\left(r^{5}\right) \\
& =2 \sum_{i, j, k, l} R_{i j, k l}(0) x^{k} x^{l} x^{i} x^{j}+O\left(r^{5}\right) .
\end{aligned}
$$

It is known that (see [2])

$$
\frac{1}{r^{4} w_{n-1}} \int_{S(r)} \sum_{i, j, k, l} R_{i j, k l}(0) x^{k} x^{l} x^{i} x^{j} d \Omega=\frac{2}{n(n+2)} \Delta R(0)
$$

and

$$
R(x)=\frac{1}{2} \sum_{i, j} R_{, i j}(0) x^{i} x^{j}+O\left(r^{3}\right),
$$


where $S(r)$ is the geodesic sphere of radius equal to $r, d \Omega$ is the volume element on the unit sphere $\mathbb{S}^{n-1} \subset \mathbb{R}^{n}$ and $w_{n-1}$ is the volume of the unit sphere $\mathbb{S}^{n-1}$. Therefore,

$$
\begin{aligned}
& \int_{B\left(0, \lambda^{\beta}\right)} \sigma_{2}\left(g_{v}\right) d \operatorname{vol}\left(g_{v}\right) \\
& =\int_{B\left(0, \lambda^{\beta}\right)} \frac{2 n(n-1) \lambda^{2}}{\left(\lambda+r^{2}\right)^{n}}+\frac{\lambda r^{2} \Delta R(0)}{2 n\left(\lambda+r^{2}\right)^{n-2}} d x \\
& \quad+\int_{B\left(0, \lambda^{\beta}\right)}\left(\frac{2 r^{4} \Delta R(0)}{n(n+2)\left(\lambda+r^{2}\right)^{n-2}}+\frac{O(r)}{\left(\lambda+r^{2}\right)^{n-4}}\right) d x \\
& =\lambda^{-\frac{n}{2}+2} \int_{B\left(0, \lambda^{\beta-1 / 2}\right)}\left(\frac{2 n(n-1)}{\left(1+r^{2}\right)^{n}}+a(x) \lambda^{2} \Delta R(0)\right) d x+O\left(\lambda^{-\frac{n}{2}+4+1 / 2}\right),
\end{aligned}
$$

where

$$
a(x)=\frac{|x|^{2}}{2 n\left(1+|x|^{2}\right)^{n-2}}+\frac{2|x|^{4}}{n(n+2)\left(1+|x|^{2}\right)^{n-2}} .
$$

Thus, (79) yields. Similarly, we can estimate

$$
\begin{aligned}
\int_{B\left(0, \lambda^{\beta}\right)} d \operatorname{vol}\left(g_{v}\right) & =\int_{B\left(0, \lambda^{\beta}\right)} v^{-n} \sqrt{\operatorname{det} g_{1}} d x \\
& =\int_{B\left(0, \lambda^{\beta}\right)} \frac{1+O\left(r^{5}\right)}{\left(\lambda+r^{2}\right)^{n}} d x \\
& =\lambda^{-\frac{n}{2}} \int_{B\left(0, \lambda^{\beta-1 / 2}\right)} \frac{d x}{\left(1+r^{2}\right)^{n}}+O\left(\lambda^{-\frac{n}{2}+5 / 2}\right) \\
& =\lambda^{-\frac{n}{2}}\left[B+O\left(\lambda^{5 / 2}+\lambda^{n(1 / 2-\beta)}\right)\right] .
\end{aligned}
$$

Therefore, we finish the proof.

LEMMA 5. - Let $g_{1}$ as above and $\gamma \in(0,2)$ be given. Assume $n \geqslant 9$. For sufficiently small $\delta>0$ such that $\lambda^{1 / 4} \gg \delta \gg \lambda^{1 / 2}$, there exist a constant $1>\delta_{1}>\delta$ and a function $u: B_{\delta_{1}} \rightarrow \mathbb{R}$ satisfying:

(0) $\delta_{1}^{\frac{n-4}{2}}=\left(\frac{2}{\gamma}-1\right) \lambda^{-1} \delta^{\frac{n}{2}}(1+o(1))$;

(1) the metric $\tilde{g}=e^{-2 u} g_{1}$ has positive $\Gamma_{2}$-curvatures;

(2) $u=\log \left(\lambda+|x|^{2}\right)+b_{0}$ for $|x| \leqslant \delta$;

(3) $u=\gamma \log |x|$ for $|x| \geqslant \delta_{1}$;

(4) $\operatorname{vol}\left(B_{\delta_{1}} \backslash B_{\delta}, \tilde{g}\right) \leqslant C\left(\delta^{\frac{n+4-n \gamma}{2(2-\gamma)}} / \lambda\right)^{2 n(2-\gamma) /(n-4)}$;

(5) $\int_{B_{\delta_{1}} \backslash B_{\delta}} \sigma_{2}(\tilde{g}) d \operatorname{vol}(\tilde{g}) \leqslant C \delta^{4+n(1-\gamma)} \lambda^{-3+2 \gamma}$, where $b_{0}$ satisfies (94) below.

Proof. - We want to find a function $u$ with $u^{\prime}(r)=\frac{\alpha(r)}{r}$. The Schouten tensor of $\tilde{g}=e^{-2 u} g_{1}$ is

$$
S(\tilde{g})_{i j}=\nabla_{i j}^{2} u+\nabla_{i} u \nabla_{j} u-\frac{|\nabla u|^{2}}{2}\left(g_{1}\right)_{i j}+S\left(g_{1}\right)_{i j}
$$




$$
=\frac{2 \alpha}{2 r^{2}} \delta_{i j}-\frac{\alpha^{2}}{2 r^{2}}\left(g_{1}\right)_{i j}+\left(\frac{\alpha^{\prime}}{r}+\frac{\alpha^{2}-2 \alpha}{r^{2}}\right) \frac{x_{i} x_{j}}{r^{2}}+S\left(g_{1}\right)_{i j}+O\left(r^{2}\right) \frac{\alpha}{r^{2}},
$$

so that

$$
S(\tilde{g})_{j}^{i}=\frac{2 \alpha-\alpha^{2}}{2 r^{2}} \delta_{i j}+\left(\frac{\alpha^{\prime}}{r}+\frac{\alpha^{2}-2 \alpha}{r^{2}}\right) \frac{x_{i} x_{j}}{r^{2}}+S\left(g_{1}\right)_{j}^{i}+O\left(r^{2}\right) \frac{\alpha}{r^{2}},
$$

since it follows from the Gauss Lemma that $\sum_{i}\left(g_{1}\right)^{i j} x_{i}=x_{j}$. We look for a function $\alpha(r) \in$ $(\gamma, 2)$ for all $r \in\left(\delta, \delta_{1}\right)$. Hence one can find a fixed constant $A>0$ independent of $\lambda$ such that

$$
S(\tilde{g})_{j}^{i} \geqslant \frac{2 \alpha-\alpha^{2}-A r^{2} \alpha}{2 r^{2}} \delta_{i j}+\left(\frac{\alpha^{\prime}}{r}+\frac{\alpha^{2}-2 \alpha}{r^{2}}\right) \frac{x_{i} x_{j}}{r^{2}}
$$

and

$$
S(\tilde{g})_{j}^{i} \leqslant \frac{2 \alpha-\alpha^{2}+A r^{2} \alpha}{2 r^{2}} \delta_{i j}+\left(\frac{\alpha^{\prime}}{r}+\frac{\alpha^{2}-2 \alpha}{r^{2}}\right) \frac{x_{i} x_{j}}{r^{2}} .
$$

Consequently, we obtain

$$
\sigma_{2}(\tilde{g}) \geqslant e^{4 u} \frac{(n-1)}{2}\left(\frac{2 \alpha-\alpha^{2}-A r^{2} \alpha}{2 r^{2}}\right)^{2}\left(n-4+4 \frac{r \alpha^{\prime}-A r^{2} \alpha}{2 \alpha-\alpha^{2}-A r^{2} \alpha}\right)
$$

and

$$
\sigma_{1}(\tilde{g}) \geqslant e^{2 u}\left(\frac{2 \alpha-\alpha^{2}-A r^{2} \alpha}{2 r^{2}}\right)\left(n-2+2 \frac{r \alpha^{\prime}-A r^{2} \alpha}{2 \alpha-\alpha^{2}-A r^{2} \alpha}\right) .
$$

We want to find an $\alpha$ satisfying

$$
\alpha= \begin{cases}\frac{2 r^{2}}{\lambda+r^{2}}, & \text { if }|x| \leqslant \delta \\ \text { solution of (89), } & \text { if }|x| \in\left(\delta, \delta_{1}\right), \\ \gamma, & \text { if }|x| \geqslant \delta_{1} .\end{cases}
$$

Such a function can be found as follows. First we solve the following equation

$$
\frac{n-4}{4}+\frac{r \alpha^{\prime}-A r^{2} \alpha}{2 \alpha-\alpha^{2}-A r^{2} \alpha}=0 .
$$

Recall this is the Bernoulli differential equation. One can find a general solution of (89) as follows

$$
\frac{1}{\alpha}=r^{\frac{n-4}{2}} e^{-\frac{n A r^{2}}{8}}\left(\int_{1}^{r} \frac{4-n}{4} \frac{1}{t^{\frac{n-2}{2}}} e^{\frac{n A t^{2}}{8}} d t+c\right) .
$$

Set

$$
H(r)=-\frac{A n}{8} \int_{1}^{r} \frac{1}{t^{\frac{n-6}{2}}} e^{\frac{n A t^{2}}{8}} d t .
$$

We have 


$$
\begin{aligned}
\alpha & =\frac{2}{1+2 a_{1} r^{\frac{n-4}{2}} e^{-\frac{n A r^{2}}{8}}+2 H(r) r^{\frac{n-4}{2}} e^{-\frac{n A r^{2}}{8}}} \\
& =\frac{2}{1+2 a_{1} r^{\frac{n-4}{2}}+2 G(r)},
\end{aligned}
$$

where

$$
G=a_{1} r^{\frac{n-4}{2}}\left(e^{-\frac{n A r^{2}}{8}}-1\right)+H(r) r^{\frac{n-4}{2}} e^{-\frac{n A r^{2}}{8}} .
$$

Here the constant $a_{1}$ is determined by

$$
\alpha(\delta)=\frac{2 \delta^{2}}{\lambda+\delta^{2}}
$$

We have the estimate

$$
a_{1}=\frac{\lambda}{2 \delta^{\frac{n}{2}}}(1+o(1))
$$

since we use the fact $\lambda^{1 / 4} \gg \delta$. Define $\delta_{1}$ by $\alpha\left(\delta_{1}\right)=\gamma$. We have

$$
\delta_{1}^{\frac{n-4}{2}}=\left(\frac{2}{\gamma}-1\right) \lambda^{-1} \delta^{\frac{n}{2}}(1+o(1))
$$

so that $1 \gg \delta_{1} \gg \delta$. Note that $n \geqslant 9$. Hence, for all $r \in\left(\delta, \delta_{1}\right)$ we have

$$
G(r)=O(1) r^{2}
$$

so that for all $r \in\left(\delta, \delta_{1}\right)$

$$
u(r)=\frac{4}{4-n} \log \left(r^{\frac{4-n}{2}}+2 a_{1}\right)+a_{2}+O\left(r^{2}\right),
$$

where

$$
a_{2}=(\gamma-2) \log \delta_{1}-\frac{4}{4-n} \log \frac{2}{\gamma}+o(1)
$$

For $r<\delta$ we have

$$
u(r)=\log \left(\lambda+r^{2}\right)+b_{0}
$$

where

(94)

$$
b_{0}=(\gamma-2) \log \delta_{1}+O(1),
$$

where we use $\delta \gg \lambda^{1 / 2}$. In view of (89), we have

$$
\left(2 \alpha-\alpha^{2}+A r^{2} \alpha\right)\left(n-4+4 \frac{r \alpha^{\prime}+A r^{2} \alpha}{2 \alpha-\alpha^{2}+A r^{2} \alpha}\right)=2 n A r^{2} \alpha=O(1) r^{2}
$$

We also have for all $r \in\left(\delta, \delta_{1}\right)$

$$
\alpha(r) \in(\gamma, 2)
$$

and

$$
2-\alpha+A r^{2}=\frac{4 a_{1} r^{\frac{n-4}{2}}}{1+2 a_{1} r^{\frac{n-4}{2}}}+O\left(r^{2}\right) .
$$

ANNALES SCIENTIFIQUES DE L'ÉCOLE NORMALE SUPÉRIEURE 
Now we can check that

$$
\begin{aligned}
& \int_{B_{\delta_{1}} \backslash B_{\delta}} \sigma_{2}(\tilde{g}) d \operatorname{vol}(\tilde{g}) \\
& \leqslant C(n) \int_{B_{\delta_{1} \backslash B_{\delta}}} e^{(4-n) u}\left(\frac{2 \alpha-\alpha^{2}+A r^{2} \alpha}{2 r^{2}}\right)^{2}\left(n-4+4 \frac{r \alpha^{\prime}+A r^{2} \alpha}{2 \alpha-\alpha^{2}+A r^{2} \alpha}\right) d \operatorname{vol}\left(g_{1}\right) \\
& \leqslant O(1) \int_{\delta}^{\delta_{1}} e^{(4-n) a_{2}}\left(r^{\frac{4-n}{2}}+2 a_{1}\right)^{4} \frac{1}{r^{2}}\left(2-\alpha+A r^{2}\right) r^{n-1} d r \\
& \leqslant O(1) \int_{\delta}^{\delta_{1}} \delta_{1}^{(n-4)(2-\gamma)} r^{5-n} a_{1} r^{\frac{n-4}{2}}\left(1+2 a_{1} r^{\frac{n-4}{2}}\right)^{4} d r \\
& \leqslant O(1) \int_{\delta}^{\delta_{1}} \delta_{1}^{(n-4)(2-\gamma)} a_{1} r^{3-\frac{n}{2}} d r \\
& \leqslant O(1) \delta_{1}^{(n-4)(2-\gamma)} \delta^{4-\frac{n}{2}} a_{1}=O(1) \delta^{n(1-\gamma)+4} \lambda^{-3+2 \gamma}
\end{aligned}
$$

and

$$
\begin{aligned}
\operatorname{vol}\left(B_{\delta_{1}} \backslash B_{\delta}, \tilde{g}\right) & =\int_{B_{\delta_{1}} \backslash B_{\delta}} e^{-n u} d \operatorname{vol}\left(g_{1}\right) \\
& \leqslant O(1) \int_{\delta}^{\delta_{1}} e^{-n a_{2}}\left(r^{\frac{4-n}{2}}+2 a_{1}\right)^{4 n /(n-4)} r^{n-1} d r \\
& =O(1) \int_{\delta}^{\delta_{1}} \delta_{1}^{n(2-\gamma)} r^{-1-n}\left(1+2 a_{1} r^{\frac{n-4}{2}}\right)^{4 n /(n-4)} d r \\
& \leqslant O(1) \delta_{1}^{n(2-\gamma)} \delta^{-n}=O(1)\left(\frac{\delta^{\frac{n+4-n \gamma}{2(2-\gamma)}}}{\lambda}\right)^{2 n(2-\gamma) /(n-4)} .
\end{aligned}
$$

Therefore, after smoothing $u$, we get a desired $u$.

We write $g_{0}=e^{-2 u_{0}} g_{1}$. In the following result, we try to connect the initial metric $g_{0}$ to some tube object. More precisely, we prove the following lemma.

Lemma 6. - Let $g_{0} \in \Gamma_{2}^{+}$and the geodesic ball $B\left(0, r_{0}\right)$ as above. Assume that $n \geqslant 5$. For any given $\gamma \in(0,2)$, then there is a conformal metric $\tilde{g}=e^{-2 u} g_{1}$ of positive $\Gamma_{2}$-curvatures on $B\left(0, r_{0}\right) \backslash\{0\}$ satisfying:

(1) The metric $\tilde{g}=e^{-2 u} g_{1}$ has positive $\Gamma_{2}$-curvatures;

(2) $u=\gamma \log |x|$ for $|x| \leqslant r_{2}$

(3) $u=u_{0}(x)+b_{1}$ for $|x| \geqslant r_{1}$;

where $r_{2}<r_{1}<r_{0}$ and $b_{1}$ is a constant.

Proof. - We write $u(x)=w(r)+\xi(r) u_{0}(x)$ where $\xi(r)$ is some cut-off function equal to 1 near $r_{0}$ and to 0 near 0 , and $w$ with $w^{\prime}(r)=\frac{\alpha(r)}{r}$, where $\alpha$ is equal to 0 near $r_{0}$. As before, the Schouten tensor of $\tilde{g}=e^{-2 u} g_{1}$ is 


$$
\begin{aligned}
S(\tilde{g})_{i j}= & \nabla_{i j}^{2} w+\nabla_{i} w \nabla_{j} w+\nabla_{i} w \nabla_{j}\left(\xi u_{0}\right)+\nabla_{i}\left(\xi u_{0}\right) \nabla_{j} w \\
& -\left(\frac{|\nabla w|^{2}}{2}+\left\langle\nabla w, \nabla\left(\xi u_{0}\right)\right\rangle\right)\left(g_{1}\right)_{i j}+S\left(e^{-2 \xi u_{0}} g_{1}\right)_{i j}
\end{aligned}
$$

so that

$$
\begin{aligned}
S(\tilde{g})_{j}^{i}= & \frac{2 \alpha-\alpha^{2}}{2 r^{2}} \delta_{i j}+\left(\frac{\alpha^{\prime}}{r}+\frac{\alpha^{2}-2 \alpha}{r^{2}}\right) \frac{x_{i} x_{j}}{r^{2}} \\
& +S\left(e^{-2 \xi u_{0}} g_{1}\right)_{j}^{i}+O\left(r+\left|\nabla\left(\xi u_{0}\right)\right|\right) \frac{\alpha}{r} .
\end{aligned}
$$

Fix $\varepsilon \in\left(0, \frac{2-\gamma}{5}\right)$ and let $C_{1}$ bound the term $O\left(r+\left|\nabla u_{0}\right|\right)$. Set $r_{4}=\min \left(\frac{r_{0}}{2}, \frac{1}{2}, \frac{\varepsilon}{2\left(1+C_{1}\right)}\right)$. For some small $r_{3}$ to be fixed later, we want $\alpha$ to decrease from $\gamma$ to 0 in $\left(r_{3}, r_{4}\right)$ and $\xi \equiv 1$ in $\left(r_{3}, r_{0}\right)$. In $B_{r_{0}} \backslash B_{r_{3}}$, we write $A=S(\tilde{g})-S\left(g_{0}\right)$. Therefore

$$
\sigma_{2}(\tilde{g})=e^{4\left(w+u_{0}\right)} \sigma_{2}\left(A+S\left(g_{0}\right)\right) .
$$

We want $A+S\left(g_{0}\right) \in \Gamma_{2}^{+}$in $B_{r_{0}} \backslash B_{r_{3}}$. It is clear in $B_{r_{4}} \backslash B_{r_{3}}$

$$
A>\left(\frac{2 \alpha-\alpha^{2}-\varepsilon \alpha}{2 r^{2}} \delta_{i j}+\left(\frac{\alpha^{\prime}}{r}+\frac{\alpha^{2}-2 \alpha}{r^{2}}\right) \frac{x_{i} x_{j}}{r^{2}}\right) .
$$

This gives

$$
\sigma_{2}(A)>e^{4 u} \frac{(n-1)}{2}\left(\frac{2 \alpha-\alpha^{2}-\varepsilon \alpha}{2 r^{2}}\right)^{2}\left(n-4+4 \frac{r \alpha^{\prime}-\varepsilon \alpha}{2 \alpha-\alpha^{2}-\varepsilon \alpha}\right)
$$

and

$$
\sigma_{1}(A)>e^{2 u}\left(\frac{2 \alpha-\alpha^{2}-\varepsilon \alpha}{2 r^{2}}\right)\left(n-2+2 \frac{r \alpha^{\prime}-\varepsilon \alpha}{2 \alpha-\alpha^{2}-\varepsilon \alpha}\right) .
$$

We see that for all small $\delta>0$,

$$
\alpha(r)=\frac{(2-5 \varepsilon) \delta}{\delta+r^{\frac{1}{2}-\frac{5}{4} \varepsilon}}
$$

solves the equation

$$
\frac{1}{4}\left(2 \alpha-\alpha^{2}-\varepsilon \alpha\right)=-r \alpha^{\prime}+\varepsilon \alpha
$$

We choose some $r_{5}<r_{4}$ and a nonincreasing function $\alpha$ in $\left(r_{5}, r_{4}\right)$ such that $\alpha\left(r_{4}\right)=0$, $\alpha\left(r_{5}\right)>0$ and $\tilde{g} \in \Gamma_{2}^{+}$in $B_{r_{4}} \backslash B_{r_{5}}$ by openness of $\Gamma_{2}^{+}$. Now we choose a suitable $\delta$ in (100) and take a small $r_{6}<r_{5}$ such that $\alpha\left(r_{6}\right)=\gamma$. From the above calculations, we see that $A \in \Gamma_{2}^{+}$ in $B_{r_{5}} \backslash B_{r_{6}}$ so that $S(\tilde{g}) \in \Gamma_{2}^{+}$in $B_{r_{5}} \backslash B_{r_{6}}$. Now we set $\alpha(r)=\gamma$ for all $r<r_{6}$ and $r_{3}=r_{6}$. We see that there exists some cut-off function $\xi$ such that $\xi(r)=1 \forall r>r_{7}, \xi(r)=0 \forall r<r_{8}$ and $r^{2} S\left(e^{-2 \xi u_{0}} g_{1}\right)$ is small in $B_{r_{7}} \backslash B_{r_{8}}$ where $r_{8}<r_{7}<r_{6}$. Thus we can choose such suitable cut-off function such that $\tilde{g}$ in $\Gamma_{2}^{+}$. Now it is sufficient to choose some $r_{2}<r_{8}$ and $r_{1}=r_{4}$. Finally, we obtain the desired $u$ by smoothing it.

The construction of such metrics of positive $\Gamma_{2}$-curvatures is motivated by the method introduced by Gromov and Lawson [14] in their study of metrics of positive scalar curvature. See also for the constructions of other positive metrics in [31,15]. Now we can prove the main result in this section. 
THEOREM 6. - Let $\left(M, g_{0}\right)$ be a compact, oriented Riemannian manifold with $g_{0} \in \Gamma_{2}^{+}$. Assume that $n \geqslant 9$. Then there exists $\tilde{g} \in\left[g_{0}\right]$ such that

$$
\tilde{g} \in \Gamma_{2}^{+}
$$

and

$$
\widetilde{\mathcal{F}}_{2}(\tilde{g})<Y_{2}\left(\mathbb{S}^{n}\right)
$$

Proof. - We fix some $\gamma \in(1,2)$ and let the geodesic ball $B\left(0, r_{0}\right)$ w.r.t. $g_{1}$ as above. We define a conformal metric $\tilde{g}$ as follows. Let $r_{2}<r_{1}<r_{0}$ as in Lemma 6 and set $\delta=\lambda^{\beta}$ with $\beta \in\left(\frac{1}{4}, \frac{1}{2}\right)$ for any small $\lambda$. Find $\delta_{1}$ as in Lemma 5 . Now for any small $\lambda$ with $\delta_{1}<r_{2}$, define $\tilde{g}$ on $B_{\delta_{1}}$ by Lemma 5 and on $B_{r_{0}} \backslash B_{r_{2}}$ by Lemma 6 . And on $M \backslash B\left(0, r_{0}\right), \tilde{g}=e^{-2 b_{1}} g_{0}$, where the constant $b_{1}$ is given in Lemma 6. Since on $B_{r_{2}} \backslash B_{\delta_{1}}$ the metrics constructed in Lemmas 5 and 6 are the same, $\tilde{g}$ is smooth. From Lemmas 5 and 6, we know (102) holds. In the following, we keep the notations of the geodesic ball with respect to the background metric $g_{1}$. By the Lemmas 4, 5 and 6, we can estimate

$$
\begin{gathered}
\int_{B_{\delta_{1}} \backslash B_{\delta}} \sigma_{2}(\tilde{g}) d \operatorname{vol}(\tilde{g}) \leqslant C \delta_{1}^{(n-4)(2-\gamma)} \delta^{4-\frac{n}{2}} a_{1}, \\
\int_{M \backslash B_{\delta_{1}}} \sigma_{2}(\tilde{g}) d \operatorname{vol}(\tilde{g}) \leqslant C \delta_{1}^{(n-4)(2-\gamma)} \delta_{1}^{4-n}, \\
\int_{B_{\delta}} \sigma_{2}(\tilde{g}) d \operatorname{vol}(\tilde{g})=\delta_{1}^{(n-4)(2-\gamma)} \lambda^{-\frac{n}{2}+2}\left[2 n(n-1) B+C \Delta R(0) \lambda^{2}\right. \\
\left.+O\left(\lambda^{5 / 2}+\lambda^{n(1 / 2-\beta)}+\lambda^{2+(n-8)(1 / 2-\beta)}\right)\right], \\
\operatorname{vol}(M, \tilde{g}) \geqslant \operatorname{vol}\left(B_{\delta}, \tilde{g}\right)=\delta_{1}^{n(2-\gamma)} \lambda^{-\frac{n}{2}}\left[B+O\left(\lambda^{5 / 2}+\lambda^{n(1 / 2-\beta)}\right)\right] .
\end{gathered}
$$

We choose some $\beta \in\left(\frac{1}{4}, \frac{n-4}{2 n}\right)$ so that we obtain

$$
\delta^{4-\frac{n}{2}} a_{1}=o\left(\lambda^{-\frac{n}{2}+4}\right) \quad \text { and } \quad \delta_{1}^{4-n}=o\left(\lambda^{-\frac{n}{2}+4}\right) .
$$

As a consequence, we get

$$
\widetilde{\mathcal{F}}_{2}(\tilde{g}) \leqslant B^{\frac{4-n}{n}}\left[2 n(n-1) B+C \Delta R(0) \lambda^{2}+o\left(\lambda^{2}\right)\right] .
$$

Recall $2 n(n-1) B^{\frac{4}{n}}=Y_{2}\left(\mathbb{S}^{n}\right)$ and $\Delta R(0)<0$. Therefore, we deduce (103) provided $\lambda$ is sufficiently small. Hence, we finish the proof.

\section{Acknowledgements}

A part of the work was carried out while the first author was visiting Max Planck Institute for Mathematics in the Sciences, Leipzig and while the second author was visiting Université Paris XII-Val de Marne. We would like to thank both institutions and Jürgen Jost and Frank Pacard for warm hospitality. The second author also would like to thank Pengfei Guan for his many helpful discussions. The main result of the paper was announced at a talk given by the second author in Magdeburg University, December 3, 2004. When this work was done, we learned that Weimin Sheng, Neil Trudinger and Xujia Wang had announced a similar result. Their result can now be 
found in [34], in which they showed that (3) is true for $n>4$. Hence, the $\sigma_{2}$-Yamabe problem is completely solved.

\section{REFERENCES}

[1] ANDrews B., Monotone quantities and unique limits for evolving convex hypersurfaces, Internat. Math. Res. Notices 1997 (1997) 1001-1031.

[2] Aubin T., Équations différentielles non linéaires et problème de Yamabe concernant la courbure scalaire, J. Math. Pures Appl. 55 (1976) 269-296.

[3] Aubin T., Li Y., On the best Sobolev inequality, J. Math. Pures Appl. 78 (1999) 353-387.

[4] Brendle S., Viaclovsky J., A variational characterization for $\sigma_{n / 2}$, Calc. Var. Partial Differential Equations 20 (2004) 399-402.

[5] Caffarelli L., Nirenberg L., Spruck J., The Dirichlet problem for nonlinear second-order elliptic equations. III. Functions of the eigenvalues of the Hessian, Acta Math. 155 (1985) 261301.

[6] Chang A., Gursky M., Yang P., An equation of Monge-Ampère type in conformal geometry, and four manifolds of positive Ricci curvature, Ann. of Math. 155 (2002) 709-787.

[7] Chang A., Gursky M., Yang P., An a priori estimate for a fully nonlinear equation on Fourmanifolds, J. Anal. Math. 87 (2002) 151-186.

[8] Chang A., Gursky M., YAng P., Entire solutions of a fully nonlinear equation, in: Lectures on Partial Differential Equations, in: New Stud. Adv. Math., vol. 2, Int. Press, Somerville, MA, 2003, pp. 43-60.

[9] CHOU K.-S., On a real Monge-Ampère functional (K.S. Tso), Invent. Math. 101 (1990) 425-448.

[10] Chou K.-S., WANG X.-J., A variational theory of the Hessian equation, Comm. Pure Appl. Math. 54 (2001) 1029-1064.

[11] Garding L., An inequality for hyperbolic polynomials, J. Math. Mech. 8 (1959) 957-965.

[12] GE Y., WANG G., On a conformal quotient equation, in preparation.

[13] GonZÁLEz M. D. M., Removability of singularities for a class of fully non-linear elliptic equations Preprint, 2004.

[14] Gromov M., LAwson H.B., The classification of simply connected manifolds of positive scalar curvature, Ann. of Math. (2) 111 (1980) 423-434.

[15] GuAn P., Lin C.-S., WANG G., Schouten tensor and some topological properties, Comm. Anal. Geom. 13 (2005) 845-860.

[16] GuAN P., Lin C.-S., WANG G., Local gradient estimates for conformal quotient equations, Preprint.

[17] Guan P., Viaclovsky J., Wang G., Some properties of the Schouten tensor and applications to conformal geometry, Trans. Amer. Math. Soc. 355 (2003) 925-933.

[18] GUAN P., WANG G., Local estimates for a class of fully nonlinear equations arising from conformal geometry, Int. Math. Res. Not. 2003 (2003) 1413-1432.

[19] GUAN P., WANG G., A fully nonlinear conformal flow on locally conformally flat manifolds, J. reine angew. Math. 557 (2003) 219-238.

[20] GuAn P., WANG G., Geometric inequalities on locally conformally flat manifolds, Duke Math. J. 124 (2004) 177-212.

[21] GUAN P., WANG G., A fully nonlinear conformal flow on locally conformally flat manifolds, math.DG/0112256, v1 of [19].

[22] GURSKY M., Viaclovsky J., Volume comparison and the $\sigma_{k}$-Yamabe problem, Adv. in Math. 187 (2004) 447-487.

[23] GURSKY M., Viaclovsky J., A fully nonlinear equation on 4-manifolds with positive scalar curvature, J. Differential Geom. 63 (2003) 131-154.

[24] GuRSKY M., ViaClovsky J., Prescribing symmetric functions of the eigenvalues of the Ricci tensor, Ann of Math., submitted for publication, math.DG/0409187.

[25] Habermann L., Riemannian Metrics of Constant Mass and Moduli Spaces of Conformal Structures, Lecture Notes in Mathematics, vol. 1743, Springer, Berlin, 2000. 
[26] Hebey E., Nonlinear Analysis on Manifolds: Sobolev Spaces and Inequalities, Courant Lecture Notes in Math., vol. 5, Courant Inst. of Math. Sci./Amer. Math. Soc., New York/Providence, RI, 1999.

[27] KRYLOV N., Nonlinear Elliptic and Parabolic Equations of the Second Order, D. Reidel, Dordrecht, 1987.

[28] Lee J., PARker T., The Yamabe problem, Bull. Amer. Math. Soc. (N.S.) 17 (1987) 37-91.

[29] Li A., Li Y., On some conformally invariant fully nonlinear equations, Comm. Pure Appl. Math. 56 (2003) 1416-1464.

[30] Lions P.L., Two remarks on the Monge-Ampère, Ann. Mat. Pura Appl. 142 (1985) 263-275.

[31] Micallef M., Wang M., Metrics with nonnegative isotropic curvature, Duke Math. J. 72 (1993) 649-672.

[32] SCHOEN R., Conformal deformation of a Riemannian metric to constant curvature, J. Differential Geom. 20 (1984) 479-495.

[33] Sha J.-P., YAng D.G., Positive Ricci curvature on the connected sums of $S^{n} \times S^{m}$, J. Differential Geom. 33 (1991) 127-137.

[34] Sheng W., Trudinger N., Wang X., The Yamabe problem for higher order curvatures, math.DG/0505463.

[35] Simon L., Asymptotics for a class of nonlinear evolution equations, with applications to geometric problems, Ann. of Math. 118 (1983) 525-571.

[36] Trudinger N., On imbeddings into Orlicz spaces and some applications, J. Math. Mech. 17 (1967) 473-483.

[37] Trudinger N., Wang X.-J., A Poincaré type inequality for Hessian integrals, Calc. Var. Partial Differential Equations 6 (4) (1998) 315-328.

[38] Viaclovsky J., Conformal geometry, contact geometry and the calculus of variations, Duke J. Math. 101 (2) (2000) 283-316.

[39] Viaclovsky J., Conformally invariant Monge-Ampère equations: Global solutions, Trans. Amer. Math. Soc. 352 (2000) 4371-4379.

[40] ViaCLOVSKY J., Estimates and some existence results for some fully nonlinear elliptic equations on Riemannian manifolds, Comm. Anal. Geom. 10 (2002) 815-847.

[41] WANG X.J., A class of fully nonlinear elliptic equations and related functionals, Indiana Univ. Math. J. 43 (1994) 25-54.

[42] YAmabe H., On a deformation of Riemannian structures on compact manifolds, Osaka Math. J. 12 (1960) 21-37.

(Manuscrit reçu le 24 mai 2005; accepté, après révision, le 16 décembre 2005.)

\footnotetext{
Yuxin GE

Laboratoire d'Analyse et de Mathématiques Appliquées, CNRS UMR 8050,

Département de Mathématiques, Université Paris XII-Val de Marne,

61, avenue du Général de Gaulle, 94010 Créteil cedex, France

E-mail: ge@univ-paris12.fr

Guofang WANG

Max-Planck-Institute for

Mathematics in the Sciences, Inselstr. 22-26,

04103 Leipzig, Germany

E-mail: gwang@mis.mpg.de
}

$4^{\text {e }}$ SÉRIE - TOME $39-2006-\mathrm{N}^{\circ} 4$ 